\title{
On a Memristor-Based Hyperchaotic Circuit in the Context of Nonlocal and Nonsingular Kernel Fractional Operator
}

\author{
Shahram Rezapour $\left(\mathbb{D},{ }^{1,2}\right.$ Chernet Tuge Deressa ${ }^{(D)},{ }^{3}$ and Sina Etemad $(1)$ \\ ${ }^{1}$ Department of Mathematics, Azarbaijan Shahid Madani University, Tabriz, Iran \\ ${ }^{2}$ Department of Medical Research, China Medical University Hospital, China Medical University, Taichung, Taiwan \\ ${ }^{3}$ College of Natural Sciences, Department of Mathematics, Jimma University, Jimma, Ethiopia
}

Correspondence should be addressed to Chernet Tuge Deressa; chernet.deressa@ju.edu.et

Received 19 August 2021; Accepted 23 September 2021; Published 25 October 2021

Academic Editor: Mehmet Emir Koksal

Copyright (c) 2021 Shahram Rezapour et al. This is an open access article distributed under the Creative Commons Attribution License, which permits unrestricted use, distribution, and reproduction in any medium, provided the original work is properly cited.

\begin{abstract}
Memristor is a nonlinear and memory element that has a future of replacing resistors for nonlinear circuit computation. It exhibits complex properties such as chaos and hyperchaos. A five-dimensional memristor-based circuit in the context of a nonlocal and nonsingular fractional derivative is considered for analysis. The Banach fixed point theorem and contraction principle are utilized to verify the existence and uniqueness of the solution of the five-dimensional system. A numerical method developed by Toufik and Atangana is used to get approximate solutions of the system. Local stability analysis is examined using the Matignon fractional-order stability criteria, and it is shown that the trivial equilibrium point is unstable. The Lyapunov exponents for different fractional orders exposed that the nature of the five-dimensional fractional-order system is hyperchaotic. Bifurcation diagrams are obtained by varying the fractional order and two of the parameters in the model. It is shown using phase-space portraits and time-series orbit figures that the system is sensitive to derivative order change, parameter change, and small initial condition change. Master-slave synchronization of the hyperchaotic system was established, the error analysis was made, and the simulation results of the synchronized systems revealed a strong correlation among themselves.
\end{abstract}

\section{Introduction}

In the last decade, fractional differential equations started gaining much attention in modeling several real-world problems in different areas including in mathematical epidemiology, physics, engineering, and many others. Fractional-order operators are either with singular kernels such as the Caputo derivative and the Reimann-Liouville fractional derivatives or with nonsingular kernels such as the Caputo-Fabrizio and Atangana-Baleanu fractional derivatives [1-4].

One of the differences between integer- and fractionalorder derivatives is that the integer-order derivative describes local properties of a certain dynamic system, whereas the fractional-order derivative representation of a dynamic system involves the whole space of the process [5]. That is, applying fractional derivative orders in modeling real-world problems is essential for describing the hereditary specifications and effectiveness of the memory as essential aspects of different mechanisms in the problem [6,7].

The recent increase in the study of different dynamical systems using fractional-order derivatives is attributed to the fact that most of the dynamic systems associated with complex systems are found to be nonlocal involving long memory in time and intrinsically fractional derivative operators can describe such systems more accurately than the integer derivatives [8]. In other words, important features of many physical systems are best described or exposed by using fractional-order operators.

There are different studies conducted using fractional derivatives for diverse types of systems pertinenet to different problems. For instance, stability analysis of fractional differential equations with unknown parameters was considered using the D-decomposition method by Koksal [9]. 
Time- and frequency-domain response of the RLC circuit with fractional-order derivative was investigated in [10]. Telegraph equation used for the power transmission line with a nonlocal boundary value problem was deliberated with a different type of finite difference numerical schemes in [11].

Chaos theory has attracted many researchers and has applications in the fields of encryption and secure of communication [12], modeling financial systems, representing circuit diagrams, and many others $[13,14]$.

Nowadays, there are many pieces of literature dedicated to the analysis of chaotic systems using fractional derivative operators. This includes chaotic systems of Chua's electrical circuits and memristor-based circuit systems. Some of the literature are reviewed as follows.

Sene applied the Caputo fractional derivative operator in detecting the chaotic behavior of different $3 \mathrm{D}$ and $4 \mathrm{D}$ chaotic systems. He used Lyapunov exponent and bifurcation diagrams to identify the nature of the chaos and impact of parameter variation for the different chaotic models investigated [15-18]. Different chaotic systems including Chua's electric circuit and several other chaotic systems were analyzed using fractional derivative order mathematical models by Petráš [19]. Bifurcation and chaotic behaviors in fractional-order simplified Lorenz system using Adams-Bashforth-Moulton predictor-corrector scheme were considered in [20]. Atangana-Baleanu fractional derivative operators were used for modeling and analysis of different chaotic and hyperchaotic systems, and solutions were approximated using a two-step Adams-Bashforth numerical scheme in [21].

It was in 1971 that circuit theorist Chua proposed memristor as a missing two-terminal nonlinear electrical component. The three basic components of a circuit are resistor, capacitor, and inductor. Memristor known for its memory effect and nonlinear characteristics is the fourth circuit component. Memristor relates magnetic flux and electric charge linkage in which case it is called a chargecontrolled electric model $(\phi=\phi(q))$ or it models a relationship between charges and flux $(q=q(\phi))$ in which case it is called a flux-controlled model $[12,22]$.

At present, there are several studies conducted on memristor-based chaotic circuits using both integer and fractional-order derivatives. In [23], a conformal fractionalorder simplest memristor-based chaotic circuit was investigated based on conformable Adomian decomposition method, Lyapunov exponent, bifurcation diagram, and Poincare sections. Buscarino et al. introduced a chaotic circuit based on a realistic model of the HP memristor, and numerical results showed a generation of chaotic attractors [22]. A novel 5D chaotic system with flux-controlled memristor and integer-order derivative, extracted from Wang's 4D hyperchaotic system, was proposed by Wang et al. [24]. A memristor-based chaotic circuit modified by replacing the nonlinear resistor in Chua's circuit with a fluxcontrolled memristor was analyzed in [12].

This research aims to study the memory effect properties and detection of chaos in a five-dimensional memristor-based system. Accordingly, an integer model memristor-based circuit is represented by Atangana-Baleanu fractional derivatives in the Caputo sense $(\mathrm{ABC})$, and the existence and uniqueness of solution of the $\mathrm{ABC}$ fractional model are analyzed based on Banach fixed point theorem for contraction principle. From the different concepts of fractional-order operators introduced above, the purpose of choosing the $\mathrm{ABC}$ fractional derivative is due to the fact that it possesses nonlocal kernels and of course it allows the inclusion of traditional initial conditions in the formulation of a mathematical model. Numerical approximation of the ABC fractional model is made using the newly developed numerical approximation for fractional derivative developed by Toufik and Atangana in [25]. Local stability of the fractional model representation is accomplished using the Matignon stability criterion. The existence and nature of chaos in the fractional model are investigated using Lyapunov exponents. A bifurcation diagram for different fractional derivatives and parameter variation is given. Several phase portraits are depicted as a verification for the impact of different parameter values and different fractional derivative orders. The impact of initial conditions on the solution trajectory of the system is also investigated using simulation of the trajectories of the system for different initial conditions. Lastly, master-slave synchronization of the system is performed accompanied by the corresponding error analysis and simulation of several cases of the synchronized system. All the phase portraits and solution trajectories in this work were obtained from the numerical scheme of Toufik and Atangana adapted for the memristor chaotic model considered in this work. A computing software application called Matlab 2019a is used for the simulation of different results.

A Matlab code for Lyapunov exponents and bifurcation diagram of fractional-order systems called Danca algorithm [26] is used to quantify the chaos by calculating Lyapunov exponents and obtaining bifurcation diagrams for different fractional orders and different parameter values of the model. Some of the evidence for the originality of this work includes the application of Atangana-Baleanu fractional operator to the memristor-based system considered in this study, application of the newly developed numerical approximation by Toufik and Atangana for the fractionalorder systems, obtaining the phase portraits of the system from the numerical scheme, and performing synchronization of the five-dimensional system using the numerical approximation.

The remaining part of this paper is arranged as follows. In Section 2, the memristor-based circuit model considered in this study is described in the context of integer derivative. Section 3 is devoted to the fractional derivative representation of the memristor-based systems following some recapping of preliminary concepts and definitions of Atangana-Baleanu fractional derivatives. The existence and uniqueness of the solution for the fractional derivative representation of the model are portrayed in Section 4 of the paper. The numerical scheme applied to get all the phase portraits and approximate time-series solution of the memristor-based system are developed in Section 5. Section 6 is concerned with the local stability analysis of the 
fractional model followed by Lyapunov exponents, bifurcation diagrams with different fractional orders, and parameter variation in Section 7. Investigation of the impact of small change in the initial conditions on the dynamics of the system is considered in Section 8 followed by synchronization of the hyperchaotic system in Section 9. Finally, in Section 10, conclusions are given followed by list of references.

\section{Mathematical Model Description of Memristor-Based Circuit}

Wang et al. [27] developed a new four-dimensional hyperchaotic circuit system using an improved modularized design and proposed implementation of the circuit. The hyperchaotic circuit system mentioned in [24] is described under an integer-order system of differential equations. Later on, Wang et al. [24] proposed a five-dimensional fluxcontrolled memristor-based circuit system and demonstrated that the system exhibits hyperchaotic character under an integer-order system of differential equations. The five-dimensional nondimensionalized flux-controlled memristor-based circuit developed by Wang et al. is described using integer-order differential as given in the following equation:

$$
\begin{aligned}
& \frac{\mathrm{d} x}{\mathrm{~d} t}=\beta_{1}(y-x)+4 y z-0.02 x(t) W(u), \\
& \frac{\mathrm{d} y}{\mathrm{~d} t}=-x+16 y-x z+w, \\
& \frac{\mathrm{d} z}{\mathrm{~d} t}=-\beta_{2} z+x y-x u-y w, \\
& \frac{\mathrm{d} w}{\mathrm{~d} t}=-10 y+0.15 x z-0.3 z u, \\
& \frac{\mathrm{d} u}{\mathrm{~d} t}=-x,
\end{aligned}
$$

where the memductance used in this study is

$$
W(u)=a+3 b u^{2}, \quad a>0, b>0 .
$$

In this study, motivated by the contribution of Wang et al. [24] in developing the five-dimensional memristorbased circuit, we analyzed system (1) under ABC fractional derivatives.

\section{Fractional Derivative Representation of the Model}

In this section, we recall the definitions and basic properties of nonsingular and nonlocal Atangana-Baleanu fractional derivative of Caputo type $(\mathrm{ABC})$ used for our analysis of the circuit.
Definition 1. Let $f \in C^{1}(a, b), a<b$, and let $q \in[0,1]$. The $\mathrm{AB}$ fractional derivative of order $q$ is defined as $[7,28,29]$

$$
{ }_{a}^{\mathrm{ABC}} D_{t}^{q} f(t)=\frac{F(q)}{1-q} \int_{a}^{t} \frac{\mathrm{d} f}{\mathrm{~d} k} E_{q}\left[-\frac{q}{1-q}(t-k)^{q}\right] \mathrm{d} k,
$$

where $F(q)=1-q-q / \Gamma(q)$ and the Mittag-Leffler function is

$$
E_{q}[z]=\sum_{\beta=0}^{\infty} \frac{z^{\beta}}{\Gamma(1+q \beta)}, \quad q, z \in \mathbb{C}, \mathfrak{R}(q)>0 .
$$

Definition 2. The Atangana-Baleanu (AB) fractional integral of the function $f \in C^{1}(a, b), a<b$, is given by $[7,28,29]$

$$
{ }_{a}^{\mathrm{AB}} I_{t}^{q} f(t)=\frac{1-q}{F(q)} f(t)+\frac{q}{F(q) \Gamma(q)} \int_{a}^{t} f(k)(t-k)^{q-1} \mathrm{~d} k
$$

Lemma 1. The $A B$ fractional derivative and $A B$ fractional integral of $f \in C^{1}(a, b), a<b$, fulfill [30]

$$
{ }_{a}^{\mathrm{AB}} I_{t}^{q}\left({ }_{a}^{\mathrm{ABC}} D_{t}^{q} f(t)\right)=f(t)-f(a) .
$$

Lemma 2. For $f, g \in C^{1}(a, b), b>a$, the $A B$ fractional derivative in the Caputo sense satisfies the Lipschitz condition [29]:

$$
\left\|\mathrm{AB}_{a}^{\mathrm{AB}} D_{t}^{q} f(t)-{ }_{a}^{\mathrm{AB}} D_{t}^{q} g(t)\right\| \leq \Lambda\|f(t)-g(t)\| .
$$

Now we continue with the reformulation of (1) in terms of $\mathrm{ABC}$ fractional derivatives.

$$
\begin{aligned}
& \text { Lemma } \begin{array}{l}
\text { 3. Let } \\
\operatorname{ABC}_{0}^{q}(f(t))=G(t), f(0)=G_{0} \text {, admits one solution given }
\end{array} \\
& \begin{array}{l}
\text { by } \\
\quad f(t)=G_{0}+\frac{1-q}{F(q)} G(t)+\frac{q}{F(q) \Gamma(q)} \int_{0}^{t}(t-k)^{q-1} G(k) \mathrm{d} k .
\end{array}
\end{aligned}
$$

We can describe the result in Lemma 3 in the form of the Banach fixed theorem point as follows. We begin by defining a Banach space

$$
X=\{x \in C(J, \mathbb{R}), J=[0,1]\},
$$

with a norm defined as $\|x\|_{x}=\operatorname{Sup}_{t \in J}|x(t)|$.

Now define the operators $M_{1}, M_{2}, M_{3}, M_{4}$, $M_{5}: X \longrightarrow X$ by 


$$
\begin{aligned}
M_{1} x(t)= & x_{0}+\frac{1-q}{F(q)}\left(\beta_{1}(y(t)-x(t))+4 y(t) z(t)-0.02 x(t) W(u(t))\right) \\
& +\frac{q}{F(q) \Gamma(q)} \int_{0}^{t}(t-k)^{q-1}\left(\beta_{1}(y-x)+4 y z-0.02 x(t) W(u(k))\right) \mathrm{d} k \\
M_{2} y(t)= & y_{0}+\frac{1-q}{F(q)}(-x(t)+16 y(t)-x(t) z(t)+w(t)) \\
& +\frac{q}{F(q) \Gamma(q)} \int_{0}^{t}(t-k)^{\alpha-1}(-x(k)+16 y(k)-x(k) z(k)+w(k)) \mathrm{d} k, \\
M_{3} z(t)= & z_{0}+\frac{1-q}{F(q)}\left(-\beta_{2} z(t)+x(t) y(t)-x(t) u(t)-y(t) w(t)\right) \\
& +\frac{q}{F(q) \Gamma(q)} \int_{0}^{t}(t-k)^{q-1}\left(-\beta_{2} z(k)+x(k) y(k)-x(k) u(k)-y(k) w(k)\right) \mathrm{d} k \\
M_{4} w(t)= & w_{0}+\frac{1-q}{F(q)}(-10 y(t)+0.15 x(t) z(t)-0.3 z(t) u(t)) \\
& +\frac{q}{F(q) \Gamma(q)} \int_{0}^{t}(t-k)^{q-1}(-10 y(k)+0.15 x(k) z(k)-0.3 z(k) u(k)) \mathrm{d} k \\
M_{5} u(t)= & z_{0}+\frac{1-q}{F(q)}(-x(t))+\frac{q}{F(q) \Gamma(q)} \int_{0}^{t}(t-k)^{q-1}(-x(k)) \mathrm{d} k .
\end{aligned}
$$

We are now ready to describe the dynamic equation for the memristor-based chaotic circuit given in (1) using ABC fractional derivative:

$$
\begin{aligned}
& { }_{0}^{\mathrm{ABC}} D_{t}^{q} x(t)=\beta_{1}(y-x)+4 y z-0.02 x(t) W(u), \\
& { }_{0}^{\mathrm{ABC}} D_{t}^{q} y(t)=-x+16 y-x z+w, \\
& { }_{0}^{\mathrm{ABC}} D_{t}^{q} z(t)=-\beta_{2} z+x y-x u-y w, \\
& { }_{0}^{\mathrm{ABC}} D_{t}^{q} w(t)=-10 y+0.15 x z-0.3 z u, \\
& { }_{0}^{\mathrm{ABC}} D_{t}^{q} u(t)=-x .
\end{aligned}
$$

The initial condition is given as $(x(0), y(0), z(0)$, $w(0), u(0))=\left(x_{0}, y_{0}, z_{0}, w_{0}, u_{0}\right)$ and $W(u)=a+3 b u^{2}$.

\section{Existence Theory on the Model}

Here, the existence and uniqueness of the solution for the $\mathrm{ABC}$ model given in (15) are shown using Banach fixed point theorem for contraction mapping. The following two theorems are worth recalling before proceeding further.
Theorem 1. Let $\Pi$ be any nonempty closed subset of a Banach space $X$. Then, any contraction $M: \Pi \longrightarrow \Pi$ has a unique fixed point [31, 32].

Theorem 2. Assume that $x, y, z, w, u$ are continuous functions satisfying the following conditions:

(C1) There exist constants $k_{1}, k_{2}, k_{3}, k_{4}, k_{5}>0$ such that $|x(t)|<k_{1},|y(t)|<k_{2},|z(t)|<k_{3},|w(t)|<k_{4},|u(t)|<k_{5}$.

(C2) $\quad(\Gamma(q)(1-q)+1)\left(\beta_{1}+0.02|W|\right)<F(q) \Gamma(q)$, $16(1-q) \Gamma(q)+16<F(q) \Gamma(q)$, and $(\Gamma(q)(1-q)+1)$ $\beta_{2}<F(q) \Gamma(q)$; then, the $A B C$ fractional derivative system given by (15) has a unique solution in the region $X$.

Proof. Let us show that the operator $M_{1}$ defined in (10) is well defined in the sense that $M_{1} x(t) \in \mathfrak{J}_{r}$ and ${ }_{0}^{\mathrm{ABC}} D_{t}^{q} M_{1} x(t)$ is continuous on $J=[0,1]$, where

$$
\mathfrak{\Im}_{r}=\{B \in X,\|B\| \leq r, r \geq 0\},
$$

for $\left(\beta_{1}\left(k_{2}+k_{1}\right)+4 k_{2} k_{3}+0.02 k_{1}\left(a+3 b k_{5}^{2}\right)\right)\left(\left\|x_{0}\right\|_{X} F(q) \Gamma\right.$ $(q)+\Gamma(q)(1-q)+1) / F(q) \Gamma(q)<r$. 
Now for any $x \in \mathfrak{\Im}_{r}$ and from (10), we have

$$
\begin{aligned}
\left\|M_{1} x\right\|= & \left\|x_{0}\right\|_{X}+\frac{1-q}{F(q)} \sup _{t \in J}\left(\beta_{1}(y(t)-x(t))+4 y(t) z(t)-0.02 x(t) W(u(t))\right) \\
& +\frac{q}{F(q) \Gamma(q)} \sup _{t \in J} \int_{0}^{t}(t-k)^{q-1}\left(\beta_{1}(y-x)+4 y z-0.02 x(t) W(u(k))\right) \mathrm{d} k \\
\leq & \left\|x_{0}\right\|+\left(\mid \beta_{1}\left(k_{2}-k_{1}\right)+4 k_{2} k_{3}-0.02 k_{1}\left(a+3 b k_{5}^{2} \mid\right)\left(\frac{1-q}{F(q)}+\frac{q}{F(q) \Gamma(q)} \sup _{t \in J} \int_{0}^{t}(t-k)^{q-1} \mathrm{~d} k\right)\right. \\
\leq & \left(\frac{\left\|x_{0}\right\| F(q) \Gamma(q)+\Gamma(q)(1-q)+1}{F(q) \Gamma(q)}\right)\left(\beta_{1}\left(k_{2}+k_{1}\right)+4 k_{2} k_{3}+0.02 k_{1}\left(a+3 b k_{5}^{2}\right)\right)<r .
\end{aligned}
$$

Consequently, we have $M_{1} x(t) \in \mathfrak{I}_{r}$.

To show continuous differentiability on $J=[0,1]$ we proceed from ${ }_{0}^{\mathrm{ABC}} D_{t}^{q} M_{1} x(t)={ }_{0}^{\mathrm{ABC}} D_{t 0}^{q \mathrm{AB}} I_{t}^{q} \quad\left(\beta_{1}(y-x)+\right.$ $4 y z-0.02 x(t) W(u)) \beta_{1}(y-x)+4 y z-0.02 x(t) W(u)$ which is continuous on $J=[0,1]$, and then we conclude that ${ }_{0}^{\mathrm{ABC}} D_{t}^{q} M_{1} x(t)$ is continuous on $J$; as a result, $M_{1} \mathfrak{\Im}_{r} \subset \mathfrak{J}_{r}$.
To show that the operator $M_{1}$ has a fixed point based on Theorem 1, it is enough to show that $M_{1}$ is a contraction mapping. Indeed, let $x_{1}, x_{2} \in X, t \in J$, and it is not difficult to show that

$$
\left\|M_{1} x_{1}-M_{1} x_{2}\right\|_{X} \leq \frac{(\Gamma(q)(1-q)+1)}{F(q) \Gamma(q)}\left(\beta_{1}+0.02\|W(u)\|_{X}\right)\left\|x_{2}-x_{1}\right\|_{X} \leq H\left\|x_{2}-x_{1}\right\|_{X}
$$

where $\quad H=(\Gamma(q)(1-q)+1)\left(\beta_{1}+0.02\|W(u)\|_{X}\right) / F(q) \Gamma$ (q). Since $H<1$ by hypothesis of Theorem 2, we conclude that $M_{1}$ is a contraction mapping.

To show that the remaining operators $M_{2}, M_{3}, M_{4}$, and $M_{4}$ are contraction mappings, we proceed in the same manner.

Let us show that the operator $M_{2}$ defined in (14) is well defined in the sense that $M_{2} y(t) \in \mathfrak{J}^{\prime}$ and ${ }_{0}^{\mathrm{ABC}} D_{t}^{q} M_{2} y(t)$ is continuous on $J=[0,1]$, where

$$
\mathfrak{\Im}_{r}^{\prime}=\left\{B \in X,\|B\| \leq r^{\prime}, r^{\prime} \geq 0\right\},
$$

for $r^{\prime}>\left(k_{1}+16 k_{2}+k_{1} k_{3}+k_{4}\right)\left(\left\|y_{0}\right\|_{X} F(q) \Gamma(q)+\Gamma(q)(1-\right.$ $q)+1) / F(q) \Gamma(q)$.

Now for any $y \in \mathfrak{\Im}_{r}^{\prime}$ and from (11), we have

$$
\begin{aligned}
\left\|M_{2} y(t)\right\|_{x}= & \left\|y_{0}\right\|_{X}+\frac{1-q}{F(q)} \sup _{t \in J}(-x(t)+16 y(t)-x(t) z(t)+w(t)) \\
& +\frac{q}{F(q) \Gamma(q)} \sup _{t \in J} \int_{0}^{t}(t-k)^{q-1}(-x(k)+16 y(k)-x(k) z(k)+w(k)) \mathrm{d} k \\
\leq & \frac{\left(k_{1}+16 k_{2}+k_{1} k_{3}+k_{4}\right)\left(\left\|y_{0}\right\|_{X} F(q) \Gamma(q)+\Gamma(q)(1-q)+1\right)}{F(q) \Gamma(q)} \leq r ı .
\end{aligned}
$$

Consequently, we have $M_{2} y(t) \in \mathfrak{\Im}_{r}^{\prime}$. To show continuous differentiability on $J=[0,1]$, we proceed from ${ }_{0}^{\mathrm{ABC}} D_{t}^{q} M_{2} y(t)={ }_{0}^{\mathrm{ABC}} D_{t 0}^{q \mathrm{AB}} I_{t}^{q}(-x+16 y-x z+w)=-x+16$ $y-x z+w$ which is continuous on $J=[0,1]$, and we conclude that ${ }_{0}^{\mathrm{ABC}} D_{t}^{q} M_{2} y(t)$ is continuous on $J$; as a result, ${ }_{0}^{\mathrm{ABC}} D_{t}^{q} M_{2} y(t)$.

To show that the operator $M_{2}$ has a fixed point, we apply Theorem 1. Following the theorem, it is sufficient to show 
that $M_{2}$ is a contraction mapping. Indeed, let $y_{1}, y_{2} \in X, t \in J$. Then,

$$
\begin{aligned}
\left\|M_{2} y_{2}-M_{2} y_{1}\right\|_{X} & \leq \frac{1-q}{F(q)} 16\left\|y_{2}-y_{1}\right\|+\frac{q 16\left\|y_{2}-y_{1}\right\|}{F(q) \Gamma(q)} \sup _{t \in J} \int_{0}^{t}(t-k)^{q-1} \mathrm{~d} k \\
& \leq \frac{16(1-q) \Gamma(q)+16}{F(q) \Gamma(q)}\left\|y_{2}-y_{1}\right\| \leq H^{\prime}\left\|y_{2}-y_{1}\right\|_{X},
\end{aligned}
$$

where $H^{\prime}=(16(1-q) \Gamma(q)+16) / F(q) \Gamma(q)$. Since $H^{\prime}<1$, we conclude that $M_{2}$ is a contraction mapping.

Let us show that the operator $M_{3}$ defined in (12) is well defined in the sense that ${ }_{0}^{\mathrm{ABC}} D_{t}^{q} M_{2} y(t)={ }_{0}^{\mathrm{ABC}} D_{t 0}^{q \mathrm{AB}} I_{t}^{q}(-x+$ $16 y-x z+w)$ and ${ }_{0}^{\mathrm{ABC}} D_{t}^{q} M_{3} z(t)$ is continuous on $J=[0,1]$, where

$$
\mathfrak{\Im}_{r^{\prime \prime}}^{\prime \prime}=\left\{u \in X,\|u\| \leq r^{\prime \prime}, r^{\prime \prime} \geq 0\right\},
$$

for $\quad r^{\prime \prime}>\left(\beta_{2} k_{3}+k_{1} k_{2}+k_{1} k_{5}+k_{2} k_{4}\right)\left(\left\|z_{0}\right\| F(q) \Gamma(q)+\Gamma(q)\right.$ $(1-q)+1) / F(q) \Gamma(q)$.

Now for any $z \in \Im_{r^{\prime \prime}}^{\prime \prime}$ and from (12), we have

$$
\begin{aligned}
\left\|M_{3} z(t)\right\|_{X}= & \left\|z_{0}\right\|_{X}+\frac{1-q}{F(q)} \sup _{t \in J}\left(-\beta_{2} z(t)+x(t) y(t)-x(t) u(t)-y(t) w(t)\right) \\
& +\frac{q}{F(q) \Gamma(q)} \sup _{t \in J} \int_{0}^{t}(t-k)^{q-1}\left(-\beta_{2} z(k)+x(k) y(k)-x(k) u(k)-y(k) w(k)\right) \mathrm{d} k \\
\leq & \left\|z_{0}\right\|_{X}+\frac{1-q}{F(q)}\left|-\beta_{2} k_{3}+k_{1} k_{2}-k_{1} k_{5}-k_{2} k_{4}\right|+\frac{q}{F(q) \Gamma(q)}\left|-\beta_{2} k_{3}+k_{1} k_{2}-k_{1} k_{5}-k_{2} k_{4}\right| \sup _{t \in J} \int_{0}^{t}(t-k)^{q-1} \mathrm{~d} k \\
\leq & \frac{\left(\beta_{2} k_{3}+k_{1} k_{2}+k_{1} k_{5}+k_{2} k_{4}\right)\left(\left\|z_{0}\right\|_{X} F(q) \Gamma(q)+\Gamma(q)(1-q)+1\right)}{F(q) \Gamma(q)}<r^{\prime \prime}
\end{aligned}
$$

Consequently, we have $M_{3} z(t) \in \mathfrak{\Im}_{r^{\prime \prime}}^{\prime \prime}$. To show continuous differentiability on $J=[0,1]$, we proceed from ${ }_{0}^{\mathrm{ABC}} D_{t}^{q} M_{3} z(t)={ }_{0}^{\mathrm{ABC}} D_{t 0}^{q \mathrm{AB}} I_{t}^{q}\left(-\beta_{2} z+x y-x u-y w\right)=-\beta_{2} z$ $+x y-x u-y w$ which is continuous on $J=[0,1]$, and we conclude that ${ }_{0}^{\mathrm{ABC}} D_{t}^{q} M_{3} z(t)$ is continuous on $J$; as a result, $M_{3} \mathfrak{\Im}_{r^{\prime \prime}}^{\prime \prime} \subset \mathfrak{\Im}_{r^{\prime \prime}}^{\prime \prime}$.
To show that the operator $M_{3}$ has a fixed point, we apply Theorem 1. Following the theorem, it is sufficient to show that $M_{3}$ is a contraction mapping. Indeed, let $z_{1}, z_{2} \in X, t \in J$. Then,

$$
\begin{aligned}
\left\|M_{3} z_{1}-M_{3} z_{2}\right\|_{X} & \leq \frac{1-q}{F(q)}\left(\beta_{2}\left\|z_{2}-z_{1}\right\|_{X}\right)+\frac{q}{F(q) \Gamma(q)} \beta_{2}\left\|z_{2}-z_{1}\right\|_{X} \sup _{t \in J} \int_{0}^{t}(t-k)^{q-1} \mathrm{~d} k \\
& \leq \frac{(\Gamma(q)(1-q)+1) \beta_{2}}{F(q) \Gamma(q)}\left\|z_{2}-z_{1}\right\|_{X} \leq H^{\prime \prime}\left\|z_{2}-z_{1}\right\|_{X}
\end{aligned}
$$

where $H^{\prime \prime}=(\Gamma(q)(1-q)+1) \beta_{2} / F(q) \Gamma(q)$. Since by hypothesis $H^{\prime \prime}<1$, we conclude that $M_{3}$ is a contraction mapping.
We have then proved that the operators $M_{1}, M_{2}$, and $M_{3}$ are well defined and are contraction mappings. The case for the operators $M_{4}$ and $M_{5}$ follows immediately. Hence, by 
the Banach fixed point theorem, system (17) has a unique solution in $X$.

\section{Numerical Solutions}

In this part, the numerical scheme applied to get the phase portrait of the dynamic system (15) is introduced. In the context of chaotic or hyperchaotic fractional differential equations, the use of analytical methods such as the Sudumu transform method, the Laplace transform method, the homotopy analysis method, and the homotopy perturbation method cannot easily be applied because of the nonlinearities of the system [13]. This leads to the need for using numerical methods to approximate the solutions of systems of fractional differential equations. Some of the numerical methods that can be applied for this case include Adams-Bashforth and Toufik-Atangana numerical schemes [8]. Both of these methods are based on Lagrange interpolation polynomials. In this study, the newly developed numerical approximation for fractional derivatives by Toufik and Atangana is employed. The numerical scheme is particularly developed for approximation of Atangana-Baleanu fractional derivative considered in this study, and it is proved to be convergent, stable, and consistent [8].

For convenience, let us write (15) in the following form:

$$
\begin{aligned}
& { }_{0}^{\mathrm{ABC}} D_{t}^{q} x=N_{1}(t, x, y, z, w, u), \\
& { }_{0}^{\mathrm{ABC}} D_{t}^{q} y=N_{2}(t, x, y, z, w, u), \\
& { }_{0}^{\mathrm{ABC}} D_{t}^{q} z=N_{3}(t, x, y, z, w, u), \\
& { }_{0}^{\mathrm{ABC}} D_{t}^{q} w=N_{4}(t, x, y, z, w, u), \\
& { }_{0}^{\mathrm{ABC}} D_{t}^{q} u=N_{5}(t, x, y, z, w, u),
\end{aligned}
$$

where

$$
\begin{aligned}
& N_{1}(t, x, y, z, w, u)=\beta_{1}(y-x)+4 y z-0.02 x(t) W(u), \\
& N_{2}(t, x, y, z, w, u)=-x+16 y-x z+w, \\
& N_{3}(t, x, y, z, w, u)=-\beta_{2} z+x y-x u-y w, \\
& N_{4}(t, x, y, z, w, u)=-10 y+0.15 x z-0.3 z u, \\
& N_{5}(t, x, y, z, w, u)=-x .
\end{aligned}
$$

Now from Lemma 3 and the first equation of (25), we have

$$
\begin{aligned}
{ }_{0}^{\mathrm{ABC}} D_{t}^{q} x(t) & =N_{1}(t, x(t), y(t), z(t), \phi(t)), \\
x(0) & =x_{0} .
\end{aligned}
$$

The solution for (27) is given as follows:

$$
\begin{aligned}
x(t)= & x_{0}+\frac{1-q}{F(q)} N_{1}(t, x(t)) \\
& +\frac{q}{F(q) \Gamma(q)} \int_{0}^{t} N_{1}(k, x(k))(t-k)^{q-1} \mathrm{~d} k .
\end{aligned}
$$

Applying Lagrange's interpolation polynomial on $\left[t_{k}, t_{k+1}\right]$ to equality $N_{1}(s, x(s), y(s), z(s), \phi(s))=\beta_{1}(y-$ $x)+4 y z-0.02 x(t) W(u)$ leads to

$$
x_{k} \approx \frac{1}{h}\left[\left(s-t_{k-1}\right) N_{1}\left(t_{k}, x\left(t_{k}\right), y\left(t_{k}\right)\right)-\left(s-t_{k}\right) N_{1}\left(t_{k-1}, x\left(t_{k-1}\right), y\left(t_{k-1}\right)\right)\right]
$$

where $h=t_{k}-t_{k-1}$.

$$
\begin{aligned}
x\left(t_{n+1}\right)= & x_{0}+\frac{1-q}{F(q)} N_{1}\left(t_{k}, x\left(t_{k}\right), y\left(t_{k}\right)\right) \\
& +\frac{q}{F(q) \Gamma(q)} \sum_{j=1}^{n}\left(\frac{N_{1}\left(t_{j}, x\left(t_{j}\right), y\left(t_{j}\right)\right)}{h} \Upsilon_{j-1}-\frac{N_{1}\left(t_{j-1}, x\left(t_{j-1}\right), y\left(t_{j-1}\right)\right)}{h} \Upsilon_{j}\right),
\end{aligned}
$$


where

$$
\begin{aligned}
\Upsilon_{j-1}= & \int_{t_{j}}^{t_{j+1}}\left(s-t_{j-1}\right)\left(t_{n+1}-s\right)^{q-1} \mathrm{~d} s=-\frac{1}{q}\left[\left(t_{j+1}-t_{j-1}\right)\left(t_{n+1}-t_{j+1}\right)^{q}-\left(t_{j}-t_{j-1}\right)\left(t_{n+1}-t_{j}\right)^{q}\right] \\
& -\frac{1}{q(q+1)}\left[\left(t_{n+1}-t_{j+1}\right)^{q+1}\left(t_{n+1}-t_{j+1}\right)^{q}-\left(t_{n+1}-t_{j}\right)^{q+1}\right], \\
\Upsilon_{j}= & \int_{t_{j}}^{t_{j+1}}\left(y-t_{j-1}\right)\left(t_{n+1}-s\right)^{q-1} \mathrm{~d} s=-\frac{1}{q}\left[\left(t_{j+1}-t_{j-1}\right)\left(t_{n+1}-t_{j+1}\right)^{q}\right] \\
& -\frac{1}{q(q+1)}\left[\left(t_{n+1}-t_{j+1}\right)^{q+1}-\left(t_{n+1}-t_{j}\right)^{q+1}\right] .
\end{aligned}
$$

Substituting $t_{j}=j h$ in (31) and (32) results in

$$
\begin{aligned}
\Upsilon_{j-1} & =\frac{h^{q+1}}{q(q+1)}\left[(n+1-j)^{q}(n-j+2+q)-(n-j)^{q}(n-j+2+2 q)\right], \\
\Upsilon_{j} & =\frac{h^{q+1}}{q(q+1)}\left[(n+1-j)^{q+1}-(n-j)^{q}(n-j+1+q)\right] .
\end{aligned}
$$

The expression (30) can be expressed in terms of (31) and (34) as shown in the following equation:

$$
\begin{aligned}
x\left(t_{n+1}\right)= & x_{0}+\frac{1-q}{F(q)} N_{1}\left(t_{n}, x\left(t_{n}\right), y\left(t_{n}\right)\right) \\
& +\frac{q}{F(q) \Gamma(q)} \sum_{j=1}^{n}\left(\left(\frac{N_{1}\left(t_{j}, x\left(t_{j}\right), y\left(t_{j}\right)\right)}{\Gamma(q+2)}\right) \times h^{q}\left[(n+1-j)^{q}(n-j+2+q)-(n-j) q(n-j+2+2 q)\right]\right. \\
& \left.-\left(\frac{N_{1}\left(t_{j-1}, x\left(t_{j-1}\right), y\left(t_{j-1}\right)\right)}{\Gamma(q+2)}\right) \times h^{q}\left[(n+1-j)^{q+1}-(n-j)^{q}(n-j+1+q)\right]\right) .
\end{aligned}
$$


Similarly, we get the following equations for the rest of the state variables:

$$
\begin{aligned}
& y\left(t_{n+1}\right)=y_{0}+\frac{1-q}{F(q)} N_{2}\left(t_{n}, x\left(t_{n}\right), y\left(t_{n}\right), z\left(t_{n}\right)\right) \\
& +\frac{q}{F(q) \Gamma(q)} \sum_{j=1}^{n}\left(\frac{N_{2}\left(t_{j}, x\left(t_{j}\right), y\left(t_{j}\right), z\left(t_{j}\right)\right)}{\Gamma(q+2)} \times h^{q}\left[(n+1-j)^{q}(n-j+2+q)-(n-j)^{q}(n-j+2+2 q)\right]\right. \\
& \left.-\frac{N_{2}\left(t_{j-1}, x\left(t_{j-1}\right), y\left(t_{j-1}\right), z\left(t_{j-1}\right)\right)}{\Gamma(q+2)} \times h^{q}\left[(n+1-j)^{q+1}-(n-j)^{q}(n-j+1+q)\right]\right), \\
& z\left(t_{n+1}\right)=z_{0}+\frac{1-q}{F(q)} N_{3}\left(t_{n}, y\left(t_{n}\right), z\left(t_{n}\right)\right) \\
& +\frac{q}{F(q) \Gamma(q)} \sum_{j=1}^{n}\left(\frac{N_{3}\left(t_{j}, y\left(t_{j}\right), z\left(t_{j}\right)\right)}{\Gamma(q+2)} \times h^{q}\left[(n+1-j)^{q}(n-j+2+q)-(n-j)^{q}(n-j+2+2 q)\right]\right. \\
& \left.-\frac{N_{3}\left(t_{j-1}, y\left(t_{j-1}\right), z\left(t_{j-1}\right)\right)}{\Gamma(q+2)} \times h^{q}\left[(n+1-j)^{q+1}-(n-j)^{q}(n-j+1+q)\right]\right) \\
& w\left(t_{n+1}\right)=w_{0}+\frac{1-q}{F(q)} N_{4}\left(t_{n}, y\left(t_{n}\right), z\left(t_{n}\right)\right) \\
& +\frac{q}{F(q) \Gamma(q)} \sum_{j=1}^{n}\left(\frac{N_{4}\left(t_{j}, y\left(t_{j}\right), z\left(t_{j}\right)\right)}{\Gamma(q+2)} \times h^{q}\left[(n+1-j)^{q}(n-j+2+q)-(n-j)^{q}(n-j+2+2 q)\right]\right. \\
& \left.-\frac{N_{4}\left(t_{j-1}, y\left(t_{j-1}\right), z\left(t_{j-1}\right)\right)}{\Gamma(q+2)} \times h^{q}\left[(n+1-j)^{q+1}-(n-j)^{q}(n-j+1+q)\right]\right) \\
& u\left(t_{n+1}\right)=u_{0}+\frac{1-q}{F(q)} N_{5}\left(t_{n}, y\left(t_{n}\right), z\left(t_{n}\right)\right) \\
& +\frac{q}{F(q) \Gamma(q)} \sum_{j=1}^{n}\left(\frac{N_{5}\left(t_{j}, y\left(t_{j}\right), z\left(t_{j}\right)\right)}{\Gamma(q+2)} \times h^{q}\left[(n+1-j)^{q}(n-j+2+q)-(n-j)^{q}(n-j+2+2 q)\right]\right. \\
& \left.-\frac{N_{5}\left(t_{j-1}, y\left(t_{j-1}\right), z\left(t_{j-1}\right)\right)}{\Gamma(q+2)} \times h^{q}\left[(n+1-j)^{q+1}-(n-j)^{q}(n-j+1+q)\right]\right) .
\end{aligned}
$$

\section{Local Stability Analysis}

In this section of the study, the local stability analysis of the fractional model represented in (25) is performed. It is known that the equilibrium points of chaotic systems are not generally stable. Some of the standard methods of stability analysis in fractional calculus are the Matignon criterion and the Laplace transform methods. In this work, the Matignon method is used for its simplicity and is most commonly used in the literature for the same purpose $[1,7,13,17]$.
The Matignon criterion is given by

$$
|\arg \lambda(J)|>\frac{q \pi}{2} 2
$$

where $J$ represents the Jacobian matrix, $\lambda(J)$ is the set of the eigenvalues of $J$, and $q$ is the fractional-order derivative. In the context of fractional derivative, an equilibrium point of (25) is said to be locally stable provided that the Matignon criterion (40) is satisfied for each of the eigenvalues of the Jacobian matrix. 
To determine if the equilibrium points of (25) are stable or not, we proceed as follows:

(i) The equilibrium points: the equilibrium points of (25) are given as $(0,0,0,0,0)$, and line equilibrium is given by $\{\alpha(0,0,0,0,1): \alpha \in \mathbb{R}\}$.

(ii) The Jacobian matrix: in this study, analysis is made regarding the trivial equilibrium point for simplicity. The parameter values used, unless otherwise mentioned, are set as $a=0.1, b=0.01$, $\beta_{1}=30$, and $\beta_{2}=8$. Thus, the Jacobian matrix of (25) evaluated at the trivial equilibrium point is given by

$$
J_{o}=\left(\begin{array}{ccccc}
-\beta_{1}-0.002 & \beta_{1} & 0 & 0 & 0 \\
-1 & 16 & 0 & 1 & 0 \\
0 & 0 & -\beta_{2} & 0 & 0 \\
0 & -10 & 0 & 0 & 0 \\
-1 & 0 & 0 & 0 & 0
\end{array}\right) .
$$

The eigenvalues of the Jacobian matrix $J$ are given by $\lambda_{1}=0, \quad \lambda_{2}=-29.3453, \quad \lambda_{4}=0.6981, \quad \lambda_{4}=0.6981$, and $\lambda_{5}=-8.0000$. To check the local stability of the trivial equilibrium point, using the Matignon criteria, we need to verify if all the eigenvalues satisfy condition (40). Indeed, $\left|\arg \left(\lambda_{1,3,4}\right)\right|=0>q \pi / 2$ which is not possible since $q \in(0,1)$. $\left|\arg \left(\lambda_{2,5}\right)\right|=3.1416=\pi>q \pi / 2$ is true for all $q \in(0,1)$. It can then be concluded that the equilibrium point $E_{\text {eqpts }}=(0,0,0)$ is locally unstable for the parameter values considered.

Furthermore, since one of the eigenvalues of the Jacobian matrix is with positive real part, it can be inferred that system (25) satisfies the necessary condition for showing the double scroll attractor [19].

\section{Lyapunov Exponents, Bifurcation, and Chaos with Different Fractional Orders of $q$ and Different Parameter Values}

In this section, the level of chaos in system (25) is quantified using the Lyapunov exponent method. Bifurcation diagrams of the system (25) related to the fractional derivative order $q$ and three of the parameters in the model named, $\beta_{1}, \beta_{2}$, and $\beta_{3}$, are depicted.

A Matlab code for Lyapunov exponents of fractionalorder systems named the Danca algorithm [26] is used to quantify the chaos by calculating Lyapunov exponents for different fractional orders of model (25). The initial conditions used in this part of the work are given by $(0.11,0.11,0.11,0.11)$. The parameter values used are similar to the ones used above for calculating the Jacobian matrix. The corresponding Lyapunov exponents (LEs) for different fractional orders $q=0.94,0.96,0.98,1.00$ are shown in Table 1.

It is then possible to conclude that the $5 \mathrm{D}$ system considered in this study is dissipative since the sum of the LEs in each column of Table 1 is negative and the system exhibits a hyperchaotic behavior since there are at least two positive LEs in each column of the table and the largest LE is positive. Moreover, Kaplan-Yorke dimension coresponding to the fractional derivatives considered in Table 1can be calculated. For instance, the dimension of two of the fractional orders is given as follows.

For $q=0.99$,

$\operatorname{dim}(\mathrm{LE})=4+\frac{15.3837+0.7298+0.005-8.4128}{|-30.6645|}=4.2511$.

$$
\begin{aligned}
\text { For } q & =0.98, \\
\operatorname{dim}(\mathrm{LE}) & =4+\frac{16.1855+0.7676+0.005-8.8488}{|-32.1742|}=4.2520 .
\end{aligned}
$$

7.1. Bifurcations due to Variation of the Fractional Order q. For obtaining bifurcation diagrams due to the variation of the fractional order $q$, the values of all the parameters are kept fixed and the order of the fractional derivative $q$ is varied in the interval $(0.8,1)$ with an increment of 0.001 . The other parameter values used in this simulation are $a=0.1, b=0.01, \beta_{1}=30$, and $\beta_{2}=8$, and the initial condition is $(0.11,0.11,0.11,0.11,0.11)$. The bifurcation diagram is shown in Figure 1.

As shown in Figure 1, when $q \in(0.8,1)$, the system is hyperchaotic. As a verification of the observation, the phase portraits corresponding to $q=0.98$ and $q=0.99$ using the numerical approximations for fractional-order systems indicated by equations (35)-(39) are shown in Figures 2 and 3, respectively. In both Figures 2 and 3, the dependence of the hyperchaotic system on the fractional derivative is observable as the two figures are seen to be different from each other for a fractional order difference of 0.01 . If we consider the trajectories in Figure 3, the orbit due to $q=0.98$ converged in less than 13.5 seconds which is not the case for $q=0.99$. That means different fractional derivative orders generate reasonably different dynamics of the system (25).

7.2. Bifurcations due to Variation of the Parameter $\beta_{1}$. The values of the parameters used are $a=0.1, b=0.01$, and $\beta_{2}=8$, the fractional derivative order is $q=0.99$, and the initial condition is $(0.11,0.11,0.11,0.11,0.11)$. The value of the parameter $\beta_{1}$ is made to vary in the interval $(29.5,30.5)$ with an increment of 0.001 . The bifurcation diagram is shown in Figure 4 . It can be observed from the diagram that system (25) exhibits significant hyperchaos throughout the interval under consideration. In support of this conclusion, some of the phase portraits of hyperchaotic system (25) are depicted in Figure 5 projected on different planes.

It can be inferred from Figures 5(a)-5(d) that the orbits seem to overlap initially, but as time increases, their divergence from each other increases. That is, as time goes, the orbit due to $\beta_{1}=30.5$ showed more contraction than the trajectory due to $\beta_{1}=30$; this is because of the sum of all the 
TABLE 1: LEs corresponding to different fractional orders of model (25) for a simulation time of $300 \mathrm{~s}$.

\begin{tabular}{lccccc}
\hline$q$ & 0.94 & 0.96 & 0.98 & 0.99 & 1 \\
\hline LE1 & 19.8323 & 17.9163 & 16.1855 & 15.3837 & 0.7298 \\
LE2 & 0.9387 & 0.8490 & 0.7676 & 0.0050 & 0.6938 \\
LE3 & 0.0050 & 0.0050 & 0.0050 & -8.4066 & -7.0050 \\
LE4 & -10.7674 & -9.7560 & -8.8353 & -30.6645 & -29.2172 \\
LE5 & -38.8609 & -35.3857 & -32.1742 & -22.9526 & -21.8497 \\
Sum of all LEs & -28.8523 & -26.3714 & -24.0496 & & \\
\hline
\end{tabular}

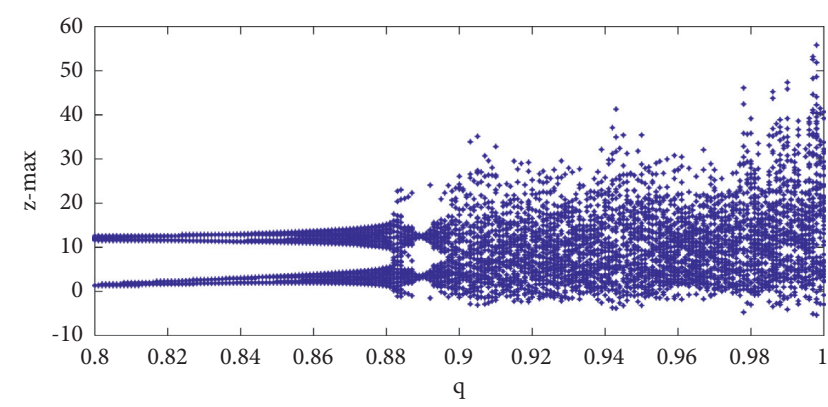

Figure 1: Bifurcation diagram for variation of the fractional derivative order $q$ in the interval $(0.8,1)$.

Lyapunov exponents (LEs) of the respective parameter values considered in this case. The sum of LEs for $\beta_{1}=30$ is -22.9526 (the LEs are shown in Table 1), the sum of LEs for $\beta_{1}=30.5$ is -23.4693 (LEs are 15.3796, 0.7298, 0.0051, -8.4066 , and -31.1772 ), and the sum of LEs for $\beta_{1}=30.25$ is -23.2106 (LEs are $15.3817,0.7298,0.0051,-8.4066$, and $-30.9209)$. Since the system under investigation is dissipative, the contraction rate of volumes due to $\beta_{1}=30.5$ is stronger than the contraction rate of volumes due to $\beta_{1}=30$ which is inferred from the magnitude of the sum of all LEs for the corresponding parameter values.

7.3. Bifurcations due to Variation of the Parameter $\beta_{2}$. The values of the parameters used are $a=0.1, b=0.01$, and $\beta_{2}=8$, the fractional derivative order is $q=0.99$, and the initial condition is $(0.11,0.11,0.11,0.11,0.11)$. The value of the parameter $\beta_{2}$ is made to vary in the interval $[7,8.5]$ with an increment of 0.001 . The bifurcation diagram is shown in Figure 6. It can be observed from the diagram that system (25) exhibits significant hyperchaos throughout the interval under consideration. In support of this conclusion, some of the phase portraits of the hyperchaotic system (25) are depicted in Figure 7 projected on different planes and some of the time-series trajectories are depicted in Figure 8.

It can be inferred from Figures 7(a)-7(c) that the orbits seem to overlap initially, but as time increases, their divergence from each other increases. That is, as time goes, the orbit due to $\beta_{1}=30.5$ showed more contraction than the trajectory due to $\beta_{1}=30$, as can be observed from the sum of all LEs of the respective parameter values considered in this case. The sum of LEs for $\beta_{2}=8$ is -22.9526 (the LEs are shown in Table 1) and the sum of LEs for $\beta_{2}=7.5$ is -22.4277 (LEs are 15.3837, 0.7298, -7.8817, 0.0050, and $-30.6645)$. Since the system under investigation is dissipative, the contraction rate of volumes due to $\beta_{2}=8$ is stronger than the contraction rate of volumes due to $\beta_{1}=7.5$ which is inferred by the magnitude of the sum of all LEs corresponding to the parameter values.

The trajectories in Figure 8 seem to overlap for the first few seconds and then begin diverging from each other; the divergence increases with time.

\section{Impact of Initial Condition}

In this section, the impact of different initial conditions on the dynamics of the system (25) is addressed. It is well known that one of the properties of chaotic systems is sensitivity to initial conditions, and thus it seems relevant to verify the impact of applying different initial conditions on the phase-space and time-series solutions of system (25). Accordingly, the parameter values and derivative order used are $a=0.1, b=0.01, \beta_{1}=30, \beta_{2}=8$, and $q=0.99$. In Figure 9 , some of the phase portraits of hyperchaotic system (25) are depicted corresponding to initial conditions $(0.11,0.11,0.11,0.11 .0 .11)$ by varying initial coordinates of $u(0)=0.5$, and 1.0 is made to run for 5 seconds with a time step of $h=0.001$. The simulation result is shown in Figure 9.

As can be observed from the figures, hyperchaotic system (25) is sensitive to changes in initial conditions. As the variation in the initial condition increases, the dynamics of the system become different from each other at least for this example.

As can be observed from Figures 9 and 10, a small difference of the initial condition generates a significantly observable change in the dynamics of system (25).

\section{Synchronization of the Hyperchaotic Model}

Synchronization between two chaotic systems is one of the most interesting phenomena in the study of dynamic systems. Synchronization is an occurrence in which two or more chaotic/hyperchaotic systems express a strong correlation among themselves. It is very interesting to see that two hyperchaotic systems, being highly sensitive to initial conditions and exponential divergence of nearby orbits, get synchronized starting from two different initial conditions. Pecora and Caroll $[33,34]$ in 1990 showed for the first time the possibility of synchronizing two chaotic systems starting from two different initial conditions. Pecora and Caroll used a technique called replacement synchronization. Chaotic synchronization has a range of applications including securing communications based on phase synchronization and controlling insulin production by beta cells. In this 


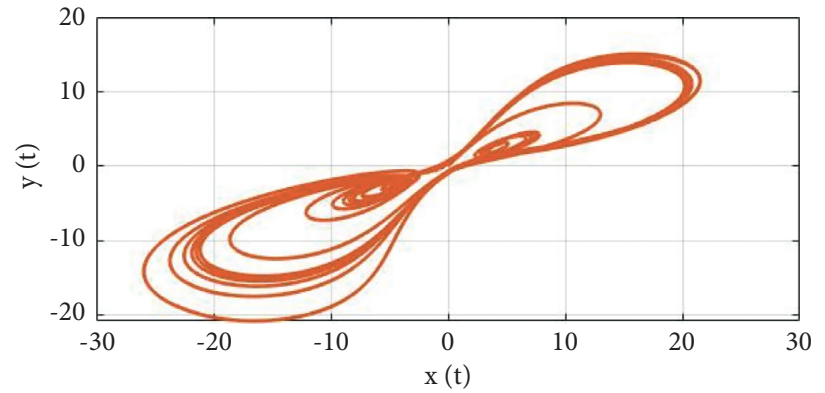

(a)

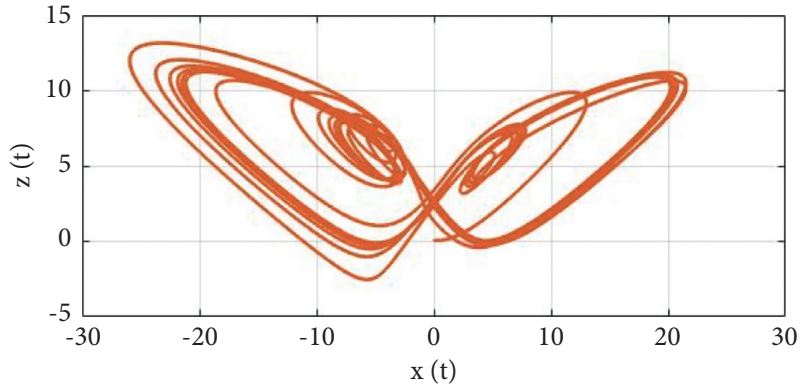

(b)

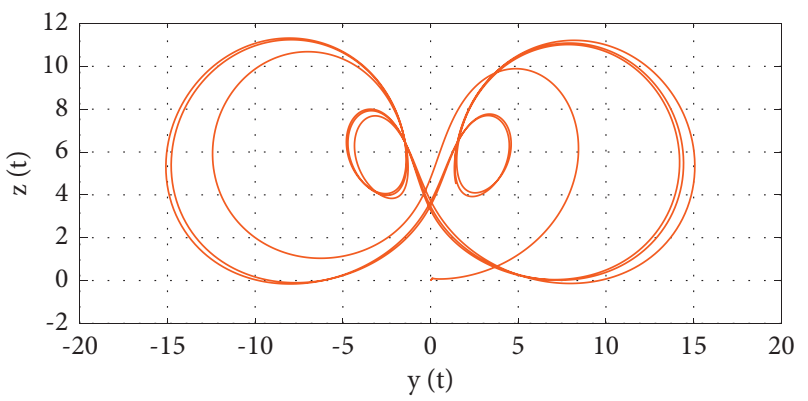

(c)

Figure 2: Phase portraits of system (25) for derivative order of $q=0.98$ projected on different planes. (a) $x-y$ plane. (b) $x-z$ plane. (c) $y-z$ plane.

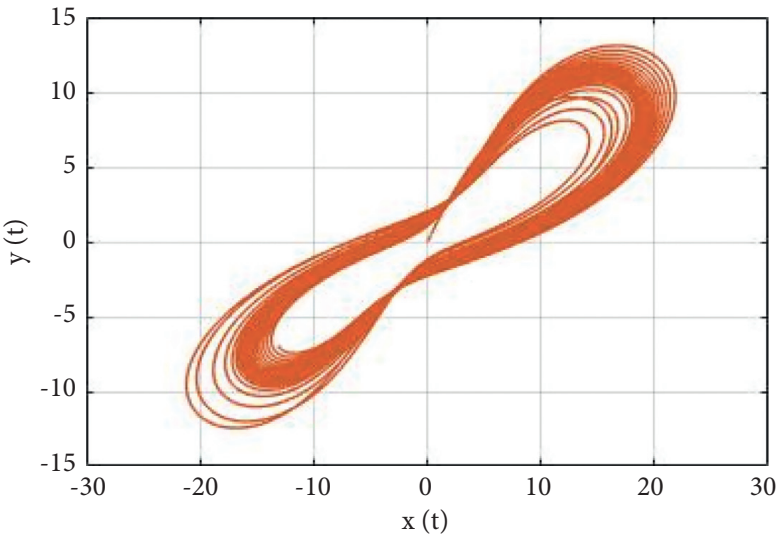

(a)

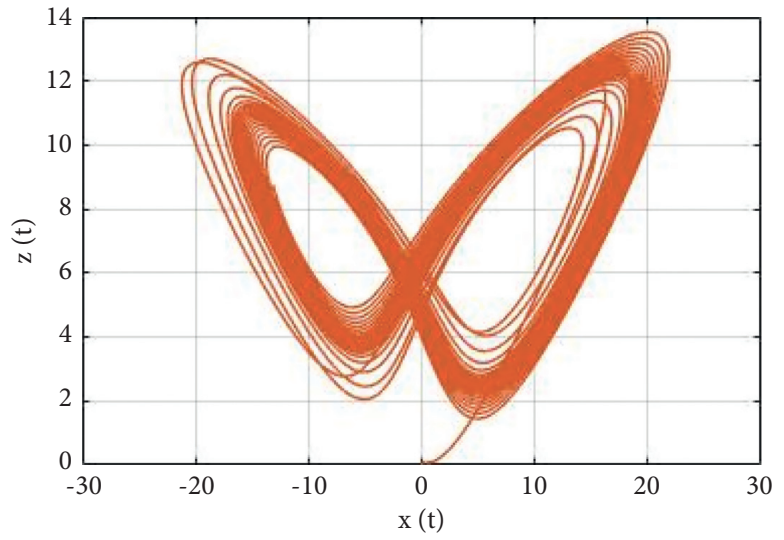

(b)

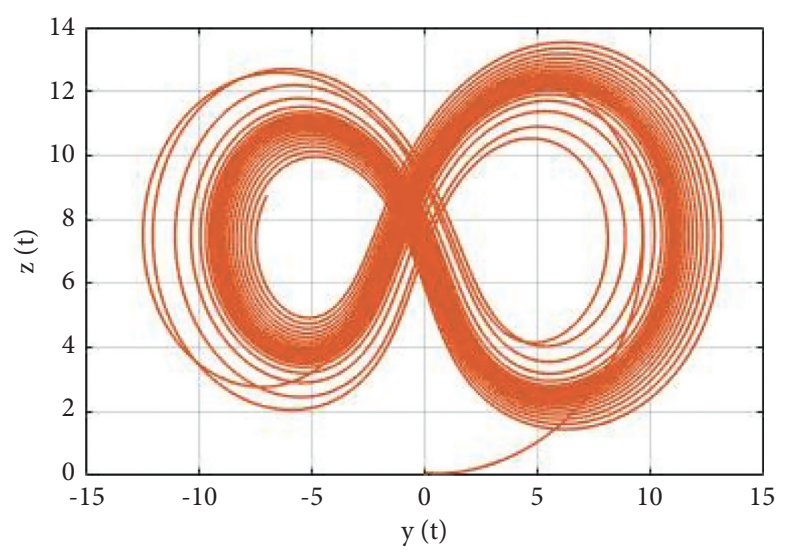

(c)

Figure 3: Phase portraits of system (25) for derivative order of $q=0.99$ projected on different planes. (a) $x-y$ plane. (b) $x-z$ plane. (c) $y-z$ plane. 


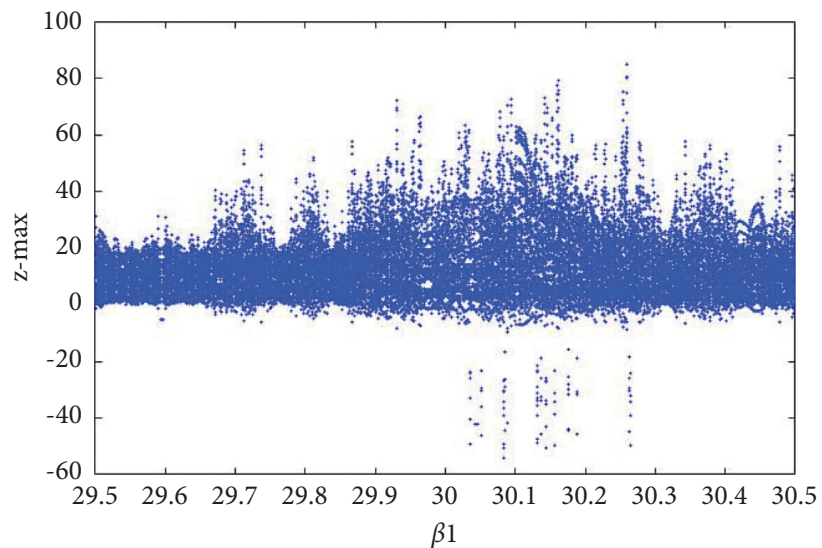

FIgURE 4: Bifurcation diagram for variation of the parameter $\beta_{1}$ in the interval $(29.5,30.5)$.

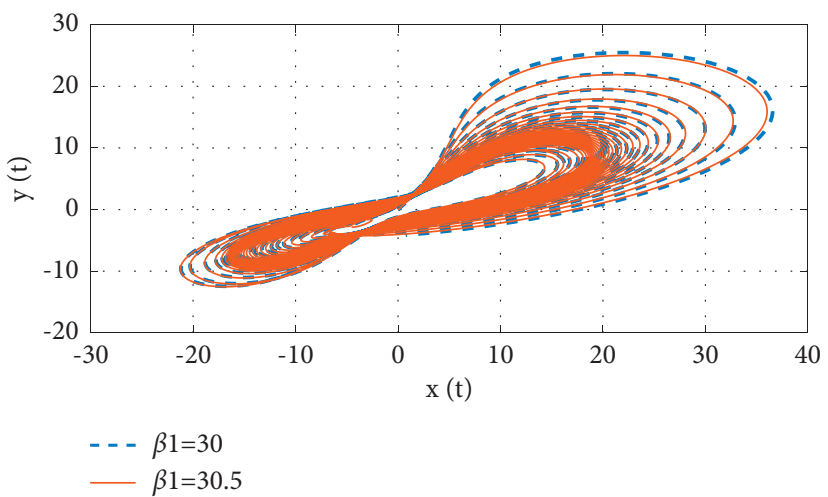

(a)

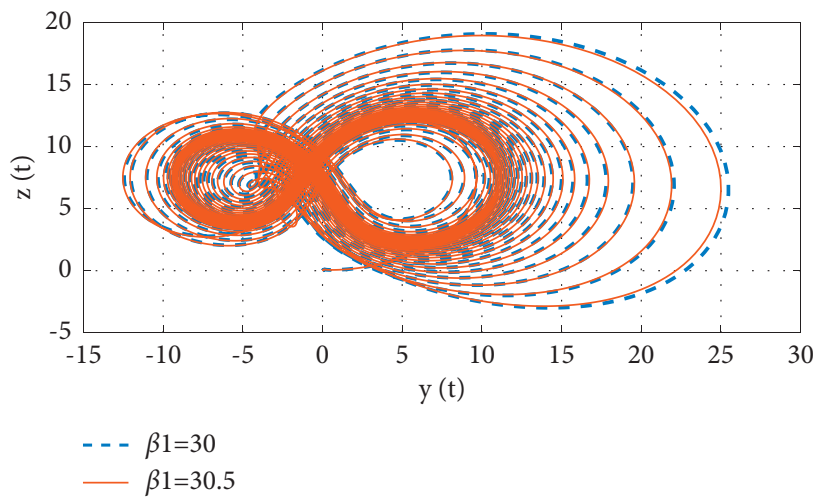

(c)

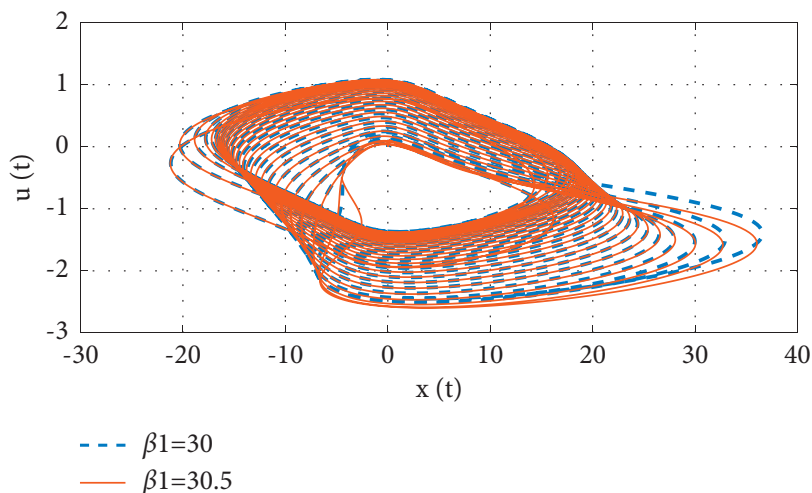

(b)

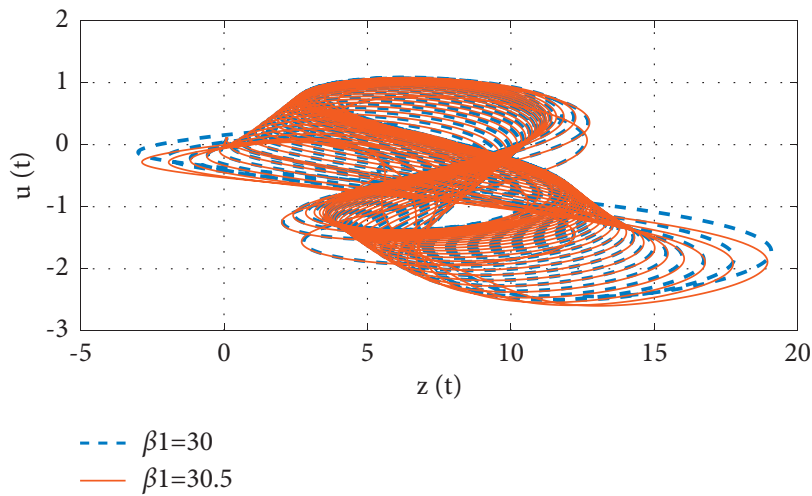

(d)

Figure 5: Phase portraits of the system (25) for different values the parameter $\beta_{1}$. (a) $x-y$ plane. (b) $x-u$ plane. (c) $y-z$ plane. (d) $z-u$ plane.

study, we applied the master-slave synchronization technique. That is, we considered two identical copies of system (25) and related them with a coupling function. We considered different initial conditions for the coupled systems, showed that the error dynamics are asymptotically stable, and portrayed some of the simulation results. The coupling function is added to the slave system to make it respond to the master system.

Now the master hyperchaotic fractional model is given by 


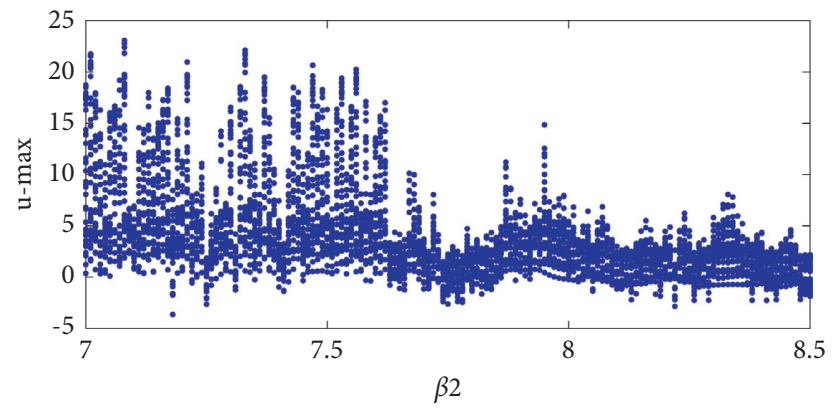

FIGURE 6: Bifurcation diagram system (25) for the parameter $\beta_{2}$ in the interval $[7,8.5]$.

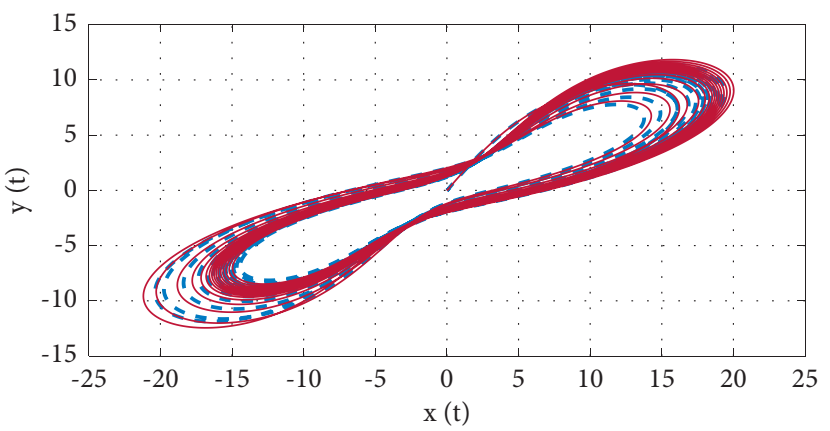

- - - $\beta 2=7.5$

$-\beta 2=8$

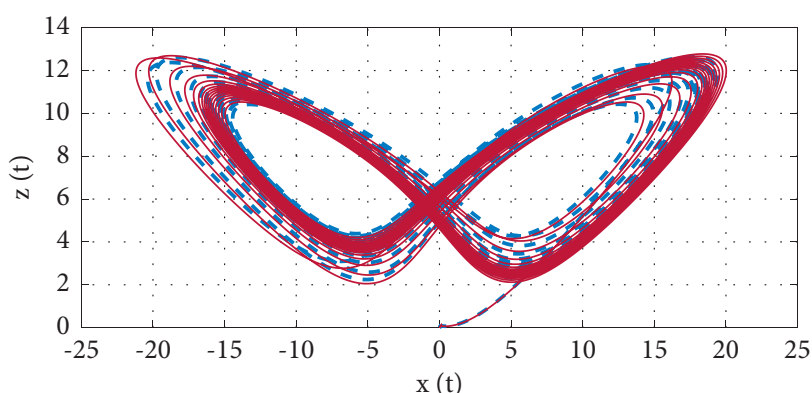

- - - $\beta 2=7.5$

$-\beta 2=8$

(a)

(b)

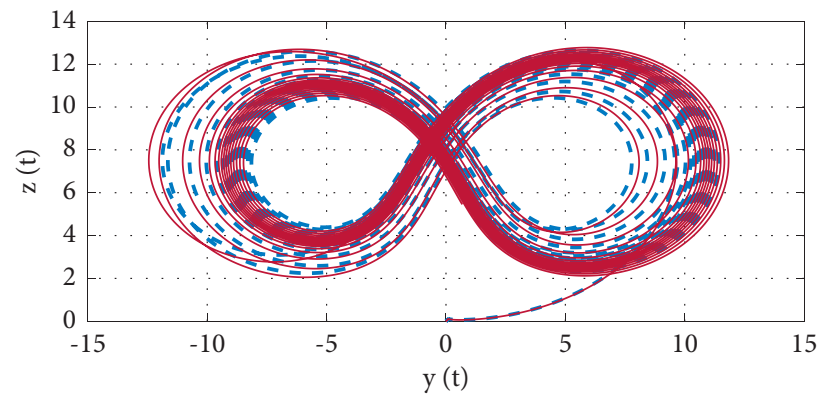

- - - $\beta 2=7.5$

$-\beta 2=8$

(c)

Figure 7: Phase portraits of the system (25) for different values parameter of $\beta_{2}$. (a) $x-y$ plane. (b) $x-z$ plane. (c) $y-z$ plane.

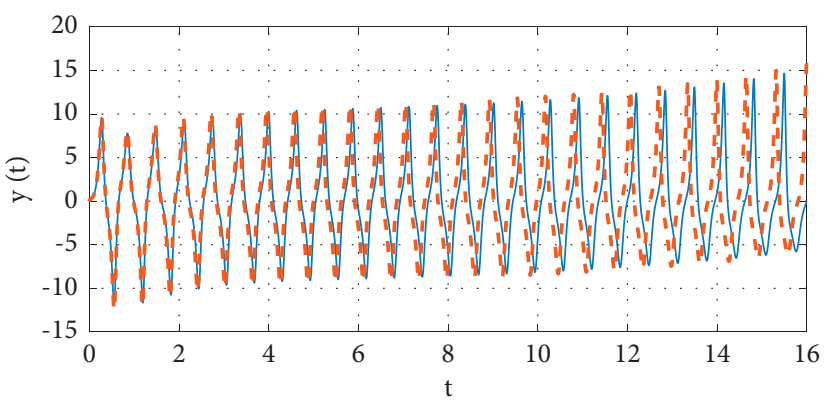

$-\beta 2=7.5$

- - - $\beta 2=8$

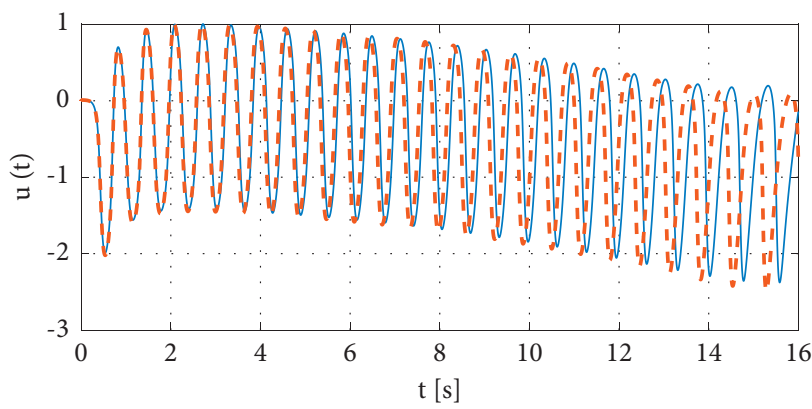

$-\beta 2=7.5$

(a)

(b)

Figure 8: Time series solutions of the state variables $y(t)$ and $u(t)$ of system (25) for different parameter values of $\beta_{2}$. 

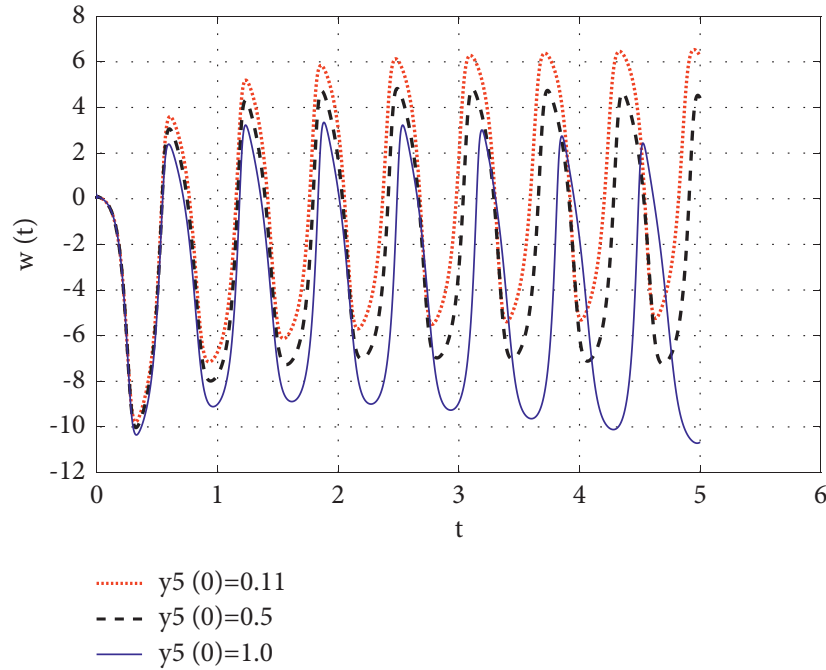

Figure $9:$

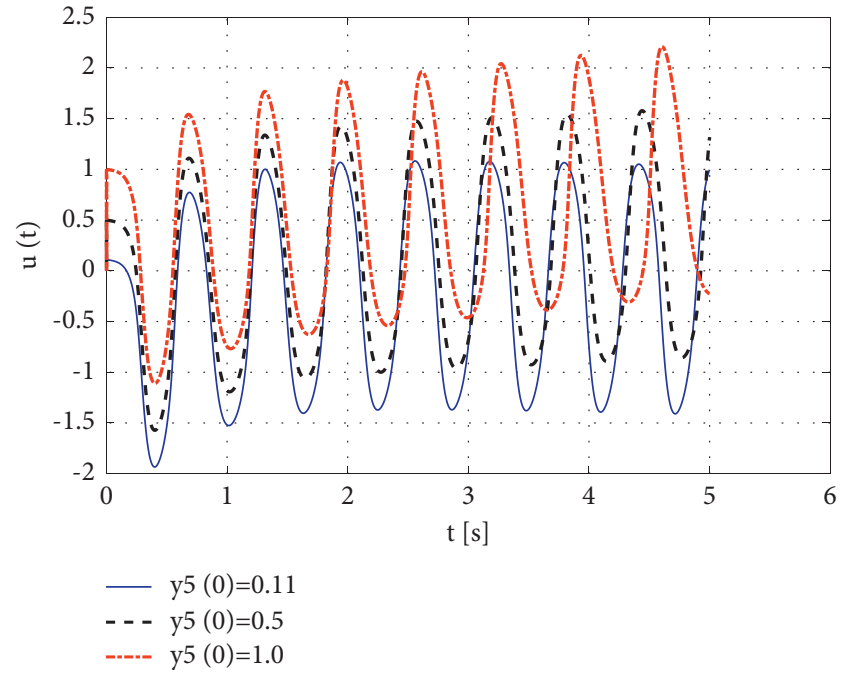

(b)

(25) due to small changes in the initial conditions.
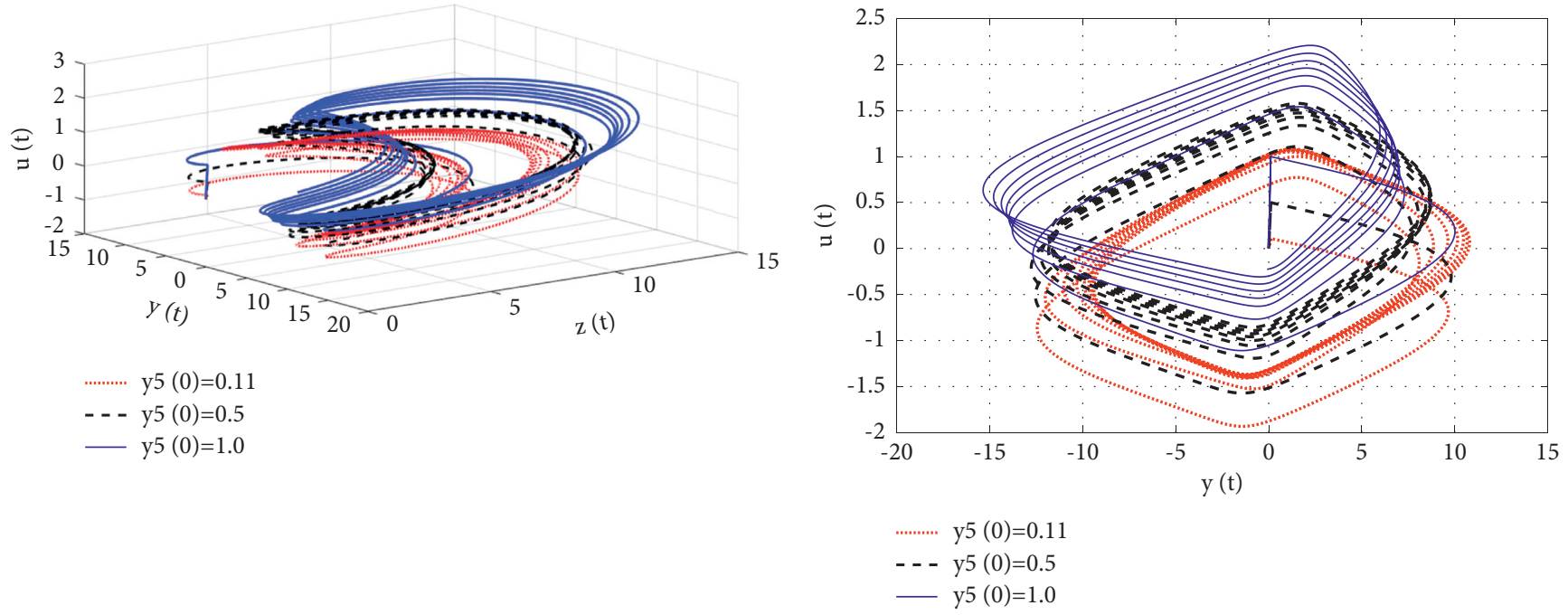

(a)

(b)

Figure 10: Some of the Phase portraits of the system (25) due to small changes in the initial condition. (a) z-y-u space. (b) y-u plane.

$$
\begin{aligned}
& { }_{0}^{\mathrm{ABC}} D_{t}^{q} x(t)=30(y-x)+4 y z-\left(0.002 x+0.0006 x u^{2}\right) \\
& { }_{0}^{\mathrm{ABC}} D_{t}^{q} y(t)=-x+16 y-x z+w \\
& { }_{0}^{\mathrm{ABC}} D_{t}^{q} z(t)=-8 z+x y-x u-y w \\
& { }_{0}^{\mathrm{ABC}} D_{t}^{q} w(t)=-10 y+0.15 x z-0.3 z u \\
& { }_{0}^{\mathrm{ABC}} D_{t}^{q} u(t)=-x
\end{aligned}
$$


Let the slave model be given by

$$
\left\{\begin{array}{l}
{ }_{0}^{\mathrm{ABC}} D_{t}^{q} x_{s}(t)=30\left(y_{s}-x_{s}\right)+4 y_{s} z_{s}-\left(0.002 x_{s}+0.0006 x_{s} u_{s}^{2}\right)+c_{1}, \\
{ }_{0}^{\mathrm{ABC}} D_{t}^{q} y_{s}(t)=-x_{s}+16 y_{s}-x_{s} z_{s}+w_{s}+c_{2}, \\
{ }_{0}^{\mathrm{ABC}} D_{t}^{q} z_{s}(t)=-8 z_{s}+y_{s} x_{s}-x_{s} u_{s}-y_{s} w_{s}+c_{3},{ }_{0}^{\mathrm{ABC}} D_{t}^{q} w_{s}(t)=-10 y_{s}+0.15 x_{s} z_{s}-0.3 u_{s} z_{s}+c_{4},{ }_{0}{ }^{\mathrm{ABC}} D_{t}^{q} u_{s}(t)=-x_{s}+c_{5} .
\end{array}\right.
$$

Define the error terms by The error dynamics are then given by $e_{1}=x_{s}-x, e_{2}=y_{s}-y, e_{3}=z_{s}-z, e_{4}=w_{s}-w, e_{5}=u_{s}-u$.

$$
\left\{\begin{array}{l}
{ }_{0}^{\mathrm{ABC}} D_{t}^{q} e_{1}(t)=\beta_{1} e_{2}-\beta_{1} e_{1}+4\left(e_{3}\left(e_{2}+y\right)+e_{2} z\right)-0.002 e_{1}+0.0006\left(-e_{1} e_{5}^{2}+2 e_{1} e_{5} u-u^{2} e_{1}-x e_{5}^{2}+2 e_{5} u x\right)+c_{1}, \\
{ }_{0}^{\mathrm{ABC}} D_{t}^{q} e_{2}(t)=-e_{1}+16 e_{2}+\left(-e_{1} e_{3}-e_{1} z-x e_{3}\right)+e_{4}+c_{2}, \\
{ }_{0}^{\mathrm{ABC}} D_{t}^{q} e_{3}(t)=-\beta_{2} e_{3}+\left(e_{1} e_{2}+e_{1} y+x e_{2}\right)+\left(-e_{1} e_{5}-e_{1} u-x e_{5}\right)+\left(-e_{2} e_{4}-e_{4} y-e_{2} w\right)+c_{3}, \\
{ }_{0}^{\mathrm{ABC}} D_{t}^{q} e_{4}(t)=-10 e_{2}+0.15\left(e_{1} e_{3}+e_{1} z+x e_{3}\right)+0.3\left(-e_{5} e_{4}-e_{5} z-u e_{4}\right)+c_{4}, \\
{ }_{0}^{\mathrm{ABC}} D_{t}^{q} e_{5}(t)=-e_{1}+c_{5} .
\end{array}\right.
$$

The vector $\left(c_{1}, c_{2}, c_{3}, c_{4}, c_{s}\right)^{T}$ is chosen as

$$
\left(\begin{array}{c}
c_{1} \\
c_{2} \\
c_{3} \\
c_{4} \\
c_{5}
\end{array}\right)=-\left(\begin{array}{c}
\beta_{1} e_{2}-\beta_{1} e_{1}+4\left(e_{3}\left(e_{2}+y\right)+e_{2} z\right)-0.002 e_{1}+0.0006\left(-e_{1} e_{5}^{2}+2 e_{1} e_{5} u-u^{2} e_{1}-x e_{5}^{2}+2 e_{5} u x\right), \\
-e_{1}+16 e_{2}+\left(-e_{1} e_{3}-e_{1} z-x e_{3}\right), \\
-\beta_{2} e_{3}+\left(e_{1} e_{2}+e_{1} y+x e_{2}\right)+\left(-e_{1} e_{5}-e_{1} u-x e_{5}\right)+\left(-e_{2} e_{4}-e_{4} y-e_{2} w\right), \\
-10 e_{2}+0.15\left(e_{1} e_{3}+e_{1} z+x e_{3}\right)+0.3\left(-e_{5} e_{4}-e_{5} z-u e_{4}\right), \\
-e_{1},
\end{array}\right)+H . \zeta
$$




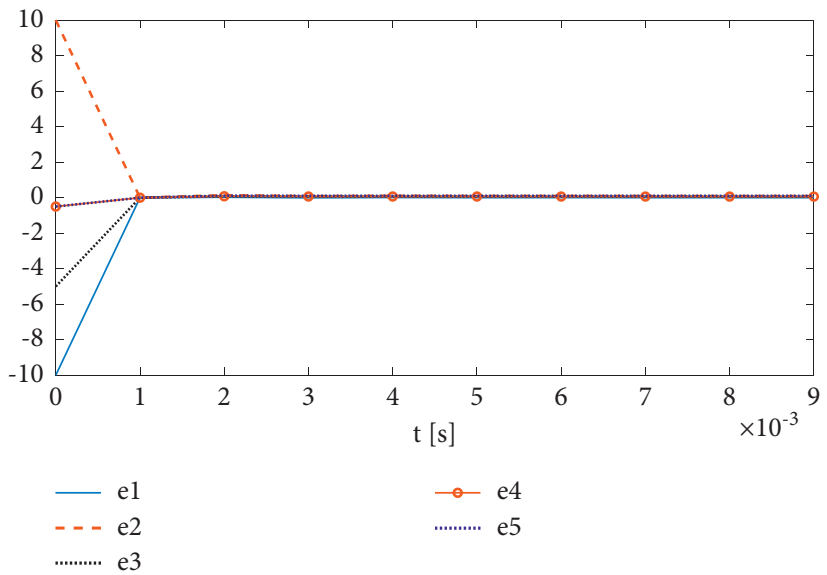

FIGURE 11: Error dynamics of the synchronization process.

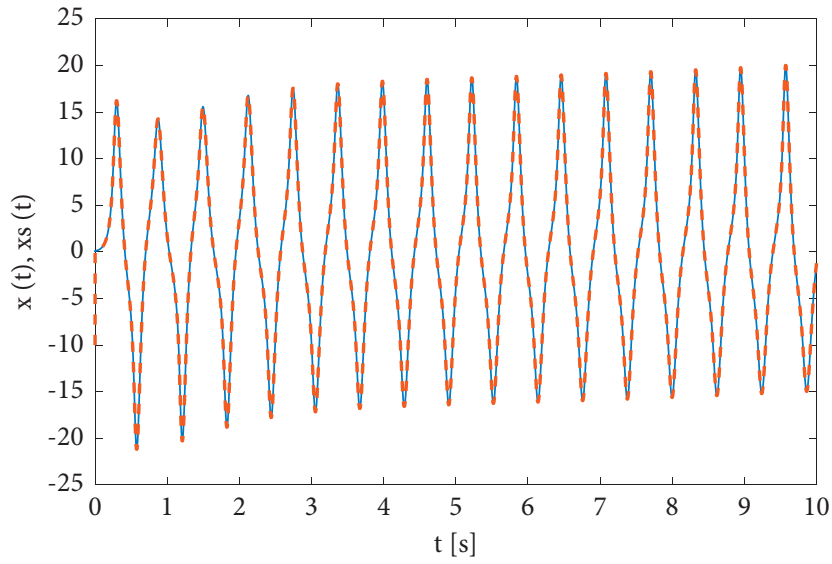

_ Master system

- - - Slave system

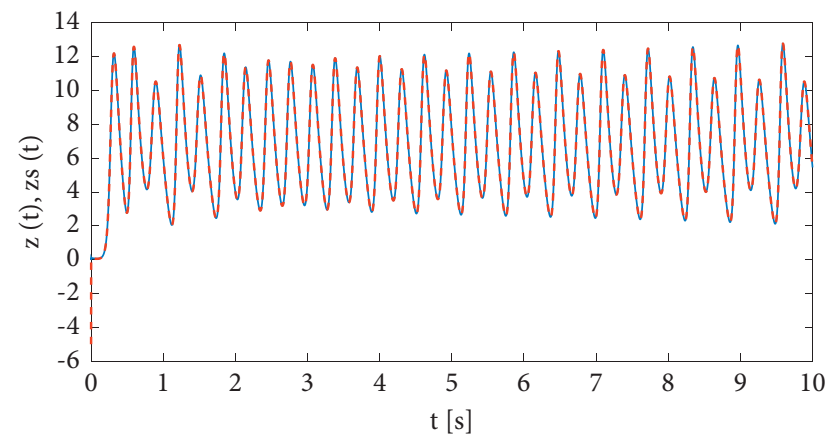

- Master system
- - Slave system

Figure 12: Some of the time series graphs of synchronized orbits. 


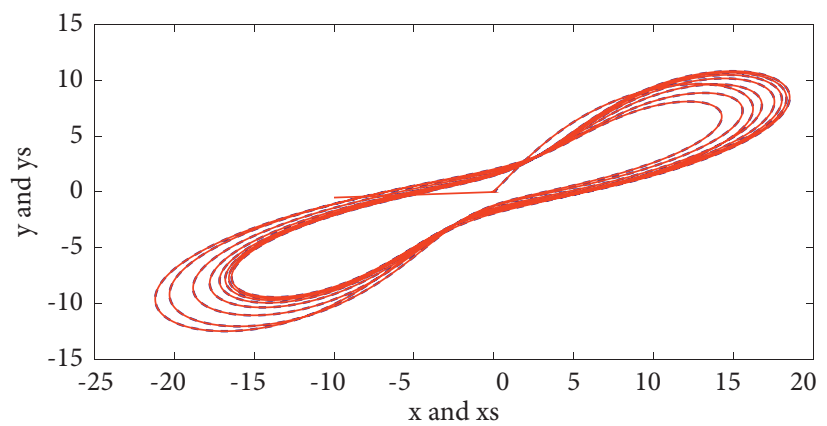

- - - Master system

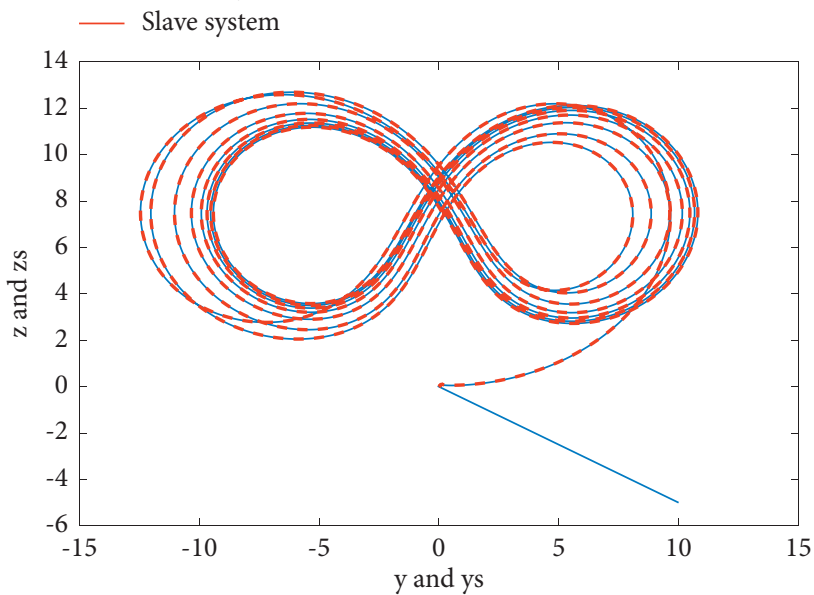

- Slave system

- - - Master system

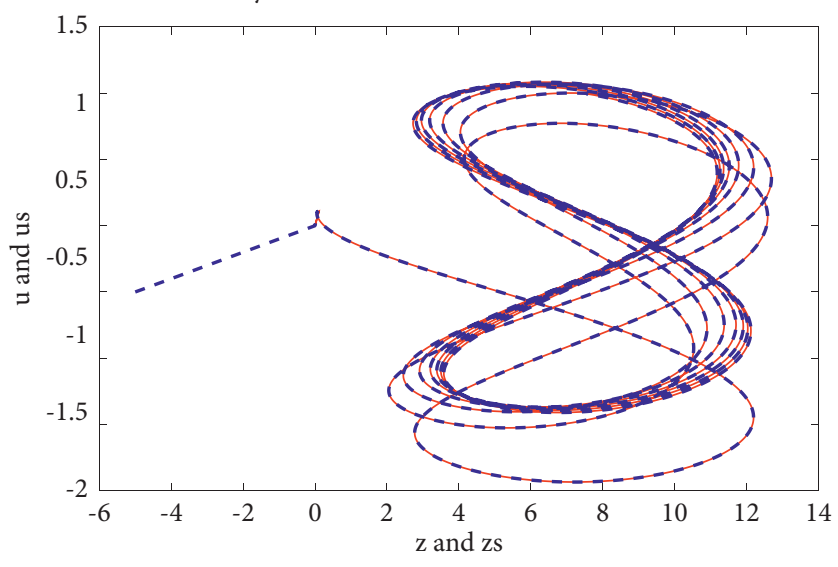

- Master system

- - - Slave system

FIGURE 13: Some of the synchronized phase spaces projected to different planes. 


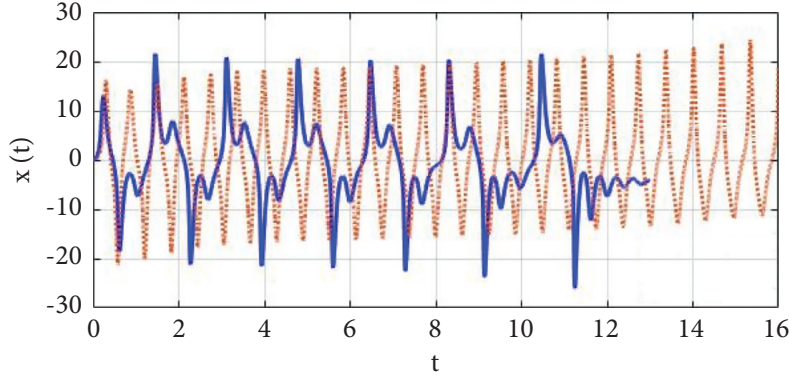

$\begin{array}{rr}\mathrm{q}=0.98 \\ \ldots . . . . & \mathrm{q}=0.99\end{array}$

(a)

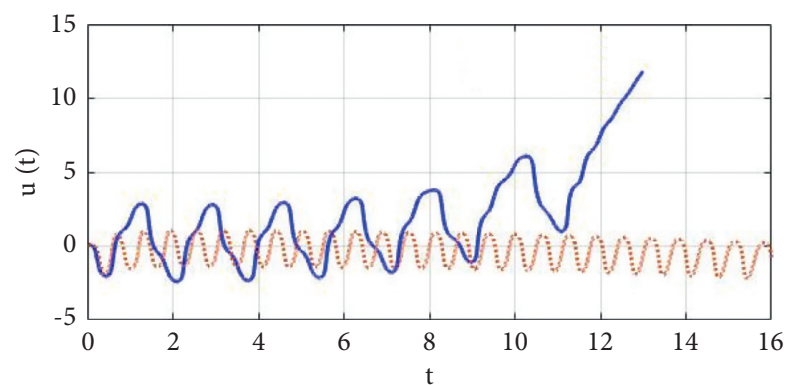

- $\mathrm{q}=0.98$

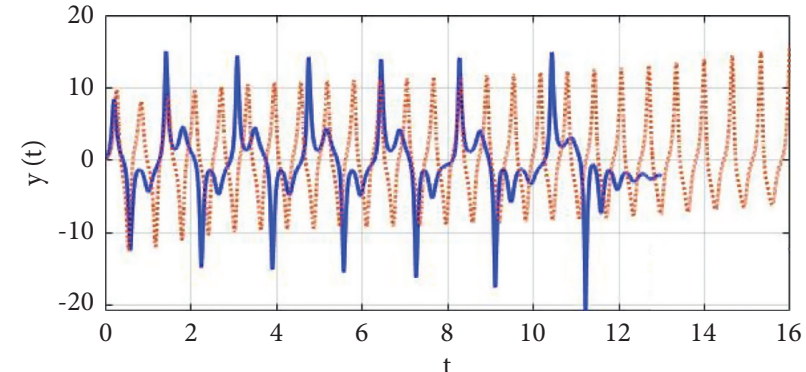

$\mathrm{q}=0.98$

$\mathrm{q}=0.99$

(b)

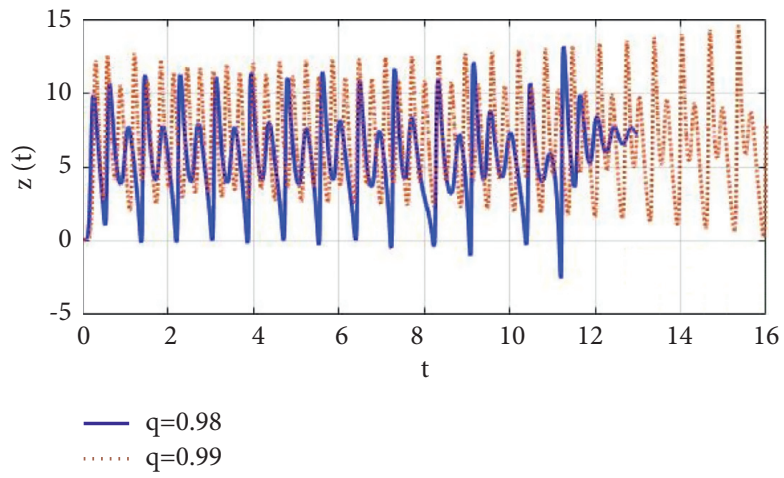

(d)

FIgURE 14: Some of the time series orbits of the system (25) due to different fractional-order derivatives.

where

$$
H . \zeta=\left(\begin{array}{ccccc}
-30 & -14.5 & 0 & 0 & 0.5 \\
-14.5 & -21 & 0 & 4.5 & 0 \\
0 & 0 & -13 & 0 & 0 \\
0 & 4.5 & 0 & -5 & 0 \\
0.5 & 0 & 0 & 0 & -5
\end{array}\right)\left(\begin{array}{l}
e_{1} \\
e_{2} \\
e_{3} \\
e_{4} \\
e_{5}
\end{array}\right) .
$$

The choice of the matrix $H$ is arbitrary except that all of its eigenvalues must satisfy the Matignon criteria of stability (40).

Then, the error dynamics become

$$
\left({ }_{0}^{\mathrm{ABC}} D_{t}^{q} e_{10}^{\mathrm{ABC}} D_{t}^{q} e_{20}^{\mathrm{ABC}} D_{t}^{q} e_{30}^{\mathrm{ABC}} D_{t}^{q} e_{40}^{\mathrm{ABC}} D_{t}^{q} e_{5}\right)=\left(\begin{array}{ccccc}
-30 & -14.5 & 0 & 0 & 0.5 \\
-14.5 & -21 & 0 & 4.5 & 0 \\
0 & 0 & -13 & 0 & 0 \\
0 & 4.5 & 0 & -5 & 0 \\
0.5 & 0 & 0 & 0 & -5
\end{array}\right)\left(\begin{array}{l}
e_{1} \\
e_{2} \\
e_{3} \\
e_{4} \\
e_{5}
\end{array}\right) .
$$

The eigenvalues of matrix $\mathrm{H}$ are $-40.8876,-13.0000$, $-12.1143,-4.9900$, and -3.0081 , and all the eigenvalues satisfy Matignon criteria, and thus the error dynamics are asymptotically stable. 
Now the slave system is given by

$$
\begin{aligned}
& { }_{0}^{\mathrm{ABC}} D_{t}^{q} x_{s}(t)=44.5 y-0.002 x+4 y z-0.5 u-0.0006 x u^{2}-30 x_{s}-14.5 y_{s}+0.5 u_{s}, \\
& { }_{0}^{\mathrm{ABC}} D_{t}^{q} y_{s}(t)=13.5 x+37 y-x z-3.5 w-14.5 x_{s}-21 y_{s}+4.5 w_{s}, \\
& { }_{0}^{\mathrm{ABC}} D_{t}^{q} z_{s}(t)=7 z+x y-x u-y w-13 z_{s}, \\
& { }_{0}^{\mathrm{ABC}} D_{t}^{q} w_{s}(t)=5 w+0.15 x z-14.5 y-0.3 z u+4.5 y_{s}-5 w_{s}, \\
& { }_{0}^{\mathrm{ABC}} D_{t}^{q} u_{s}(t)=-1.5 x+5 u+0.5 x_{s}-5 u_{s} .
\end{aligned}
$$

The numerical simulation that verified a strong correlation between the master and the slave system in the form of phase space, time-series orbits, and the error dynamics are depicted in Figures 11-13. The numerical approximation for fractional order system (25) given in equations (35)-(39) is adapted to simulate each of the error dynamics (50), the master system (44), and slave system (51). The simulation results are shown in Figures 11-13. The parameter values and derivative order used are $a=0.1, b=0.01, \beta_{1}=30, \beta_{2}=8$, and $q=0.99$. The initial condition used for master systems is $(0.11,0.11,0.11,0.11,0.11)$, and it is $(-10,10,-5,-0.5,-0.5)$ for the slave system. The error graphs indicate fast synchronization of the slave and master systems.

\section{Conclusion}

In this study, a 5-dimensional memristor-based hyperchaotic circuit in the context of the fractional operator was considered and different qualitative and quantitative analyses are made: numerical approximation of the solution, bifurcation diagrams, Lyapunov exponents for different fractional orders, and parameter values are used to investigate the nature of the solution and chaotic system. The system is found to be hyperchaotic. Sensitivity to initial conditions and sensitivity to parameter value changes were found to cause a significant effect on the dynamics of the system. Furthermore, in the course of making several simulation results on the dynamics of the system, the hyperchaotic system is sensitive to almost any change made in the system including time scale increment change used in the study. The sensitivity of the system to fractional derivative order is very strong in the sense that the simulation results for $q=0.98$ and 0.99 are significantly different (see Figures 2, 3, and 14). This is perhaps related to the contraction rate of volumes due to the different fractional-order derivatives. The magnitude of the sum of all the LEs corresponding to different fractional-order derivatives decreases as the order of the derivative increases for the case of the hyperchaotic system considered in this study (see Table 1). This requires further investigation. Synchronization of the system is also established and exhibited a strong agreement. The dependence of synchronization on the fractional derivative orders and variation of parameters needs further investigation.

\section{Data Availability}

No datasets were generated or analyzed during the current study.

\section{Conflicts of Interest}

The authors declare that they have no conflicts of interest.

\section{Authors' Contributions}

The authors declare that the study was realized in collaboration with equal responsibility. All authors read and approved the final manuscript.

\section{Acknowledgments}

The first and third authors would like to thank Azarbaijan Shahid Madani University.

\section{References}

[1] N. Sene, "Generalized Mittag-Leffler input stability of the fractional-order electrical circuits," IEEE Open Journal of Circuits and Systems, vol. 1, pp. 233-242, 2020.

[2] H. Mohammadi, S. Kumar, S. Rezapour, and S. Etemad, "A theoretical study of the Caputo-Fabrizio fractional modeling for hearing loss due to Mumps virus with optimal control," Chaos, Solitons \& Fractals, vol. 144, Article ID 110668, 2021.

[3] D. Baleanu, A. jajarmi, H. Mohammadi, and S. Rezapour, "A new study on the mathematical modelling of human liver with Caputo-Fabrizio fractional derivative," Chaos, Solitons \& Fractals, vol. 134, Article ID 109705, 2020.

[4] D. Baleanu, S. Etemad, and S. Rezapour, "A hybrid Caputo fractional modeling for thermostat with hybrid boundary value conditions," Boundary Value Problems, vol. 2020, no. 1, Article ID 64, 2020.

[5] T. Li and Y. Wang, "Stability of a class of fractional-order nonlinear systems," Discrete Dynamics in Nature and Society, vol. 2014, Article ID 724270, 2014.

[6] H. Khan, J. F. Gómez-Aguilar, A. Alkhazzan, and A. Khan, “A fractional order HIV-TB coinfection model with nonsingular Mittag-Leffler Law," Mathematical Methods in the Applied Sciences, vol. 43, no. 6, pp. 3786-3806, 2020.

[7] C. T. Deressa and G. F. Duressa, "Analysis of AtanganaBaleanu fractional-order SEAIR epidemic model with optimal 
control," Advances in Difference Equations, vol. 2021, Article ID 174, 25 pages, 2021.

[8] A. H. Assi, Engineering Education and Research Using MATLAB, Intech Open, London, UK, 2011.

[9] M. E. Koksal, "Stability analysis of fractional differential equations with unknown parameters," NAMC, vol. 24, no. 2, pp. 224-240, 2019, https://www.journals.vu.lt/nonlinearanalysis/article/view/12899.

[10] M. Emir Koksal, "Time and frequency responses of non-integer order RLC circuits," AIMS Mathematics, vol. 4, no. 1, pp. 64-78, 2019.

[11] M. E. Koksal, M. Senol, and A. K. Unver, "Numerical simulation of power transmission lines," Chinese Journal of Physics, vol. 59, pp. 507-524, 2019.

[12] Y. Li, X. Huang, and M. Guo, "The generation, analysis, and circuit implementation of a new memristor based chaotic system," Mathematical Problems in Engineering, vol. 2013, Article ID 398306, 2013.

[13] A. Atangana and K. M. Owolabi, "New numerical approach for fractional differential equations," Mathematical Modelling of Natural Phenomena, vol. 13, no. 1, pp. 1-21, 2018.

[14] N. Sene, "Study of a fractional-order chaotic system represented by the Caputo operator," Complexity, vol. 2021, Article ID 5534872, 2021.

[15] N. Sene, "Analysis of a fractional-order chaotic system in the context of the Caputo fractional derivative via bifurcation and Lyapunov exponents," Journal of King Saud University Science, vol. 33, no. 1, Article ID 101275, 2021.

[16] N. Sene, "Introduction to the fractional-order chaotic system under fractional operator in Caputo sense," Alexandria Engineering Journal, vol. 60, no. 4, pp. 3997-4014, 2021.

[17] N. Sene, "Qualitative analysis of class of fractional-order chaotic system via bifurcation and Lyapunov exponent notions," Journal of Mathematics, vol. 2021, Article ID 5548569, 2021.

[18] N. Sene, "Mathematical views of the fractional Chua's electrical circuit described by the Caputo-Liouville derivative," Revista Mexicana de Física, vol. 67, no. 1, pp. 91-99, 2021.

[19] I. Petras, Fractional-order Nonlinear Systems: Modeling, Analysis and Simulation, Springer-Verlag, Berlin, Heidelberg, 2011.

[20] K. Sun, X. Wang, and J. C. Sprott, "Bifurcations and chaos in fractional-order simplified Lorenz system," International Journal of Bifurcation and chaos, vol. 20, no. 4, pp. 1209-1219, 2010.

[21] K. M. Owolabi and A. Atangana, "On the formulation of Adams-Bashforth scheme with Atangana-Baleanu- Caputo fractional derivative to model chaotic problems," Chaos (Woodbury, N.Y.), vol. 29, no. 2, Article ID 023111, 2019.

[22] A. Buscarino, L. Fortuna, M. Frasca, and L. V. Gambuzza, "A chaotic circuit based on Hewlett-Packard memristor," Chaos (Woodbury, N.Y.), vol. 22, Article ID 023136, 2012.

[23] J. Ruan, K. Sun, J. Mou, S. He, and L. Zhang, "Fractional-order simplest memristor-based chaotic circuit with new derivative," The European Physical Journal Plus, vol. 133, Article ID 3, 2018.

[24] R. Wang, M. Li, Z. Gao, and H. Sun, "A new memristor-based $5 \mathrm{D}$ chaotic system and circuit implementation," Complexity, vol. 2018, Article ID 6069401, 2018.

[25] M. Toufik and A. Atangana, "New numerical approximation of fractional derivative with non-local and nonsingular kernel: application to chaotic models," The European Physical Journal Plus, vol. 132, no. 10, p. 444, 2017.
[26] M. F. Danca and N. Kuznetsov, "Matlab code for Lyapunov exponents of fractional-order systems," International Journal of Bifurcation and Chaos, vol. 28, no. 05, Article ID 1850067, 2018.

[27] W. Rui, S. Hui, W. Jie-Zhi, W. Lu, and W. Yan-Chao, "Applications of modularized circuit designs in a new hyperchaotic system circuit implementation," Chinese Physics B, vol. 24, no. 2, Article ID 020501, 2015.

[28] A. Atangana and S. İğret Araz, "New numerical method for ordinary differential equations: Newton polynomial," Journal of Computational and Applied Mathematics, vol. 372, Article ID 112622, 2020.

[29] A. Atangana and D. Baleanu, "New fractional derivatives with nonlocal and non-singular kernel: theory and application to heat transfer model," Thermal Science, vol. 20, no. 2, pp. 763-769, 2016.

[30] T. Abdeljawad and D. Baleanu, "Integration by parts and its applications of a new nonlocal fractional derivative with Mittag-Leffler nonsingular kernel," Journal of Nonlinear Sciences and Applications, vol. 10, no. 03, pp. 1098-1107, 2017.

[31] N. Sene and A. Ndiaye, "On class of fractional-order chaotic or hyperchaotic systems in the context of the Caputo fractional-order derivative," Journal of Mathematics, vol. 2020, Article ID 8815377, 2020.

[32] A. Granas and J. Dugundji, "Elementary fixed point theorems," in Fixed Point TheorySpringer, New York, NY, USA, 2003.

[33] L. M. Pecora and T. L. Carroll, "Synchronization in chaotic systems,” Physical Review Letters, vol. 64, no. 8, pp. 821-824, 1990.

[34] S. H. Strogatz, Nonlinear Dynamics and Chaos (With Applications to Physics, Biology, Chemistry, and Engineering), p. 498, Addison-Wesley, Reading, MA, USA, 1994. 